\title{
Non-reservation price equilibria and consumer search
}

\author{
Maarten C.W. Janssen a,b,*, Alexei Parakhonyak ${ }^{c}$, \\ Anastasia Parakhonyak ${ }^{\mathrm{d}}$ \\ a Department of Economics, University of Vienna, Austria \\ ${ }^{\mathrm{b}}$ National Research University Higher School of Economics, Moscow, Russia \\ ${ }^{\mathrm{c}}$ Department of Economics, University of Oxford, United Kingdom \\ d Toulouse School of Economics, France
}

Received 19 October 2015; final version received 7 June 2017; accepted 7 August 2017

Available online 18 August 2017

\begin{abstract}
Reservation price equilibria (RPE) do not accurately assess market power in consumer search markets. In most search markets, consumers do not know important elements of the environment in which they search (such as, for example, firms' cost). We argue that when consumers learn when searching, RPE suffer from theoretical issues, such as non-existence and critical dependence on specific out-of-equilibrium beliefs. We characterize equilibria where consumers rationally choose search strategies that are not characterized by a reservation price. Non-RPE always exist and do not depend on specific out-of-equilibrium beliefs. Non-RPE have active consumer search and are consistent with recent empirical findings.

(c) 2017 The Authors. Published by Elsevier Inc. This is an open access article under the CC BY license (http://creativecommons.org/licenses/by/4.0/).

JEL classification: D40; D83; L13

t5 This paper has benefited from presentations at the III and IV Workshops on consumer search and switching cost (HSE, Moscow 2012 and 2013), ESEM 2013, EARIE 2014, ESWC 2015, RES 2016, and the Theoretische Ausschuss (Basel, 2016) and research seminars in Vienna, Toulouse, Berlin, Mannheim, Belfast, Copenhagen, Leuven, Lancaster, Monash University, Nottingham, Oxford, Southern Methodist University and University of Technology Sydney, and from discussions with H. Bester, A. Fishman, S. Lauermann, E. Moen, J.-L. Moraga, M. Rauh, A. Rhodes, S. Shelegia, A. Suvorov and M. Wildenbeest. We also would like to thank an associate editor and two anonymous reviewers for their constructive and insightful comments. Janssen acknowledges financial support from the Austrian Science Foundation FWF, under project number P 27995-G27.

* Corresponding author.

E-mail addresses: maarten.janssen@univie.ac.at (M.C.W. Janssen), aleksei.parakhonyak@economics.ox.ac.uk (A. Parakhonyak), aparakhonyak@gmail.com (A. Parakhonyak).
\end{abstract}


Keywords: Sequential search; Non-reservation price equilibria; Asymmetric information; Consumer learning

\section{Introduction}

In consumer search markets, firms have market power due to the fact that some consumers do not make price comparisons. Firms take this market power into account when deciding on price. This paper addresses the question of whether, by focusing on consumers following reservation price strategies, the existing consumer search literature accurately evaluates this market power due to search frictions. A reservation price strategy is a cut-off strategy: after observing a price at or below some critical value, consumers decide to buy, otherwise they continue to search.

In markets where there is uncertainty about the underlying factors determining firms' pricing behavior, there are important theoretical reasons to consider other search strategies than reservation price strategies. Rothschild (1974) drew attention to the fact that when consumers do not know from which distribution of offers they obtain their information, the optimal consumer search rule may well be different from the typical reservation price rule. ${ }^{1}$ The main reason is that on the basis of past search observations, consumers learn about the environment in which they search. Depending on the environment, it may well be that, after observing a relatively good outcome, consumers infer that even better outcomes are likely to be observed in the next search round and rationally conclude to continue to search, whereas, after observing a relatively bad outcome, consumers infer that better outcomes are unlikely and thus stop searching.

The consumer search literature has, by and large, neglected this observation. The celebrated models by Stahl (1989) and Wolinsky (1986), and much of the literature that takes these models as a starting point, study environments without underlying uncertainty and in such theoretical environments the optimal search rule is indeed a reservation price rule. In consumer search markets where consumers are uninformed about firms' underlying costs (and this probably comprises most markets where consumer search is important), learning is an important part of the search process. There are some papers on learning and consumer search that take consumer uncertainty about firms' costs into consideration (see, Benabou and Gertner, 1993; Dana, 1994; Fishman, 1996 and more recently, Yang and Ye, 2008; Tappata, 2009; Janssen et al., 2011 and Chandra and Tappata, 2011). The observations by Rothschild (1974) are of immediate concern to these environments, but the relevant economics literature has continued to focus on equilibria where the consumer search rule is characterized by a reservation price.

Some of this literature is inspired by retail gasoline markets where the common wholesale price of crude oil is the most important determinant of the (variation in) costs of retailers, and consumers are uncertain about these costs due to the large fluctuations of this wholesale price on the world market. Although our focus in this paper is on consumer search in retail markets, the issues we address are also relevant for other markets. For example, Benabou and Gertner (1993) is motivated by macroeconomic concerns about inflationary uncertainty and the consequences for firms' mark-ups, while a recent paper by Duffie et al. (2017) considers over-the-counter (OTC) financial markets and the role of benchmarks in these markets. The current paper is also relevant for the labor search literature where workers search for a better wage. In labor markets, it is natural that the wage distribution depends on the business cycle and that firms are better informed about the business cycle than workers. In that case, workers learn about the wage distribution while searching for another job and their search behavior does not need to follow a reservation

\footnotetext{
1 Dubra (2004) studies how optimism and over confidence affect search.
} 
wage strategy. In all these markets, there is uncertainty and asymmetric information about a common component that determines the distribution of offers and one needs to understand how the uninformed side (consumers, workers) search and simultaneously learn in such an environment.

Our paper is the first to systematically incorporate Rothschild's observations on nonreservation price strategies into an equilibrium search model with endogenous firm behavior. ${ }^{2,3}$ Benabou and Gertner (1993) also mention the fact that in their model reservation price equilibria (RPE) may not exist. They set up the equations that have to be satisfied in a non-RPE. They perform some numerical analysis for some parameter values, but they neither have an analysis characterizing these non-RPE, nor do they show the conditions under which these equilibria exist. ${ }^{4}$

The literature studying RPE in environments where consumers are uninformed about firms' cost is unsatisfactory for a number of reasons. First, RPE are known to exist only if the search cost is relatively large and/or the uncertainty about costs is relatively small (cf., Dana, 1994 and Janssen et al., 2011). It is unclear what type of equilibria do exist for small search cost or large uncertainty about common costs. Second, RPE implicitly assume certain out-of-equilibrium beliefs and it is unknown whether these out-of-equilibrium beliefs satisfy game theoretic refinement concepts commonly employed in asymmetric information games. Third, one would expect that when costs are uncertain consumers may engage in active costly search in equilibrium. When consumers observe a high price, they are uncertain about whether this is due to a relatively high (common) production costs or whether this particular firm is charging a high margin. RPE in these homogeneous goods markets have firms charging prices below the consumer reservation price, however, and therefore all consumers buy at the first firm they visit. This lack of consumer search gives firms substantial market power, but it may well be that RPE overestimate the true market power because they underestimate consumers' search intensity.

In response to these points, this paper first sharpens existing results on RPE. We show (i) that independent of out-of-equilibrium beliefs, RPE do not exist when the uncertainty about production cost is relatively large and (ii) that an RPE, even if it exists, is sensitive to the specification of out-of-equilibrium beliefs and do not satisfy, for example, the logic of the D1 equilibrium refinement (hereafter the D1 logic, see Cho and Sobel, 1990). If the uncertainty about cost is relatively large, any equilibrium should have active search. We then continue to characterize non-RPE and show that they exist for all parameter values and that there are parameter values for which multiple non-RPE exist. Thus, non-RPE resolve the non-existence problem that RPE suffer from. Moreover, in any non-RPE, consumers actively search beyond the first firm. In particular, there is a region of "high" prices that are set with positive probability such that consumers are indifferent between buying and searching and consumers continue to search with strictly positive

\footnotetext{
2 Even though our paper focuses on consumer search, similar considerations apply to the labor search literature that uses reservation wage equilibrium as a solution concept (see McCall, 1970 for pioneering work in this direction, and subsequent literature as, for example, surveyed in Rogerson et al., 2005).

3 Lauermann et al. (2017) consider a bargaining and matching environment with uncertainty about the relative scarcity of a commodity. They also do not restrict themselves to studying reservation price strategies, but their set-up is different as they have one side of the market competing in an auction to acquire the good from the other side of the market, instead of markets where firms post prices (as in our setting).

4 Benabou and Gertner (1993, p. 74) state that the "non-reservation price equilibria (if they exist) are too complicated for us to solve" and argue that these equilibria are "somewhat less appealing intuitively than the previous reservation price equilibria" (p. 81) as they think the reservation price property is "required in particular for demand functions to be downward sloping" (p. 74). This is, however, only partially the case. In order to make firms indifferent over the range of prices in a mixed strategy equilibrium, a firm's expected total demand must be downward sloping. In the non-RPE in our model, the demand of an individual consumer must have a downward sloping part and may have an upward sloping part.
} 
probability. When the cost uncertainty is large, market prices may be substantially below the market prices predicted by RPE due to active search by consumers. On the other hand, when cost uncertainty is small, expected market prices are larger in non-RPE. Thus, whether or not RPE overestimate the market power of firms depends on the uncertainty about cost.

In a recent empirical paper, De los Santos et al. (2012) show that, when buying books online consumers do not follow reservation price strategies. These strategies predict that (i) consumers buy from the last store visited unless all stores have been visited and (ii) the decision whether to continue to search depends on the outcome of the previous search with consumers observing lower prices deciding to buy, and consumers observing larger prices deciding to continue searching. Their evidence contradicts these predictions. ${ }^{5}$ In this paper, we show that their empirical findings are consistent with equilibrium behavior under non-reservation price strategies, as follows. When the cost uncertainty is relatively large, non-RPE have a region of intermediate prices where the probability of a sale is lower when the price is low. At lower prices, consumers rationally expect to get lower prices on the next search round and this may induce them to search more. In particular, we show that consumers may accept higher prices in the first search round, while rejecting lower prices. In an extension to oligopolistic markets, we also show that the optimal sequential search behavior of consumers is consistent with consumers going back to previously sampled firms, before they have sampled all firms. ${ }^{6}$

There is also a relationship with the marketing oriented literature on reference price effects (see, e.g., Putler, 1992; Kalyanaram and Winer, 1995 and Mazumdar et al., 2005). This literature points to the fact that consumers have particular pricing points around which consumer demand is very sensitive to price changes. This may lead to situations where consumer demand drops significantly if firms price above this reference point, whereas at higher prices, consumers are willing to buy again. Such "reference point" demand behavior can occur in non-RPE when the cost uncertainty is large. After observing intermediate prices above the "reference price", consumers rationally infer that these prices are not chosen by high cost firms. Knowing costs are low, consumers find these prices too high to buy, however, and they continue to search for sure. This inference creates a gap in the equilibrium price distribution of the low cost firms.

The rest of the paper is organized as follows. Section 2 describes the model and the equilibrium concept. Section 3 discusses how RPE depend on assumptions regarding out-of-equilibrium beliefs and why (regardless of these out-of-equilibrium beliefs) they do not exist when cost uncertainty is relatively large. Section 4 describes our analytical results on non-RPE. Section 5 shows, by means of a numerical analysis, the effects of cost uncertainty on profits, expected prices and consumer welfare. Section 6 briefly discusses a generalization of our model to the case of imperfectly correlated production costs and oligopoly markets with $N$ firms. We show that with three or more firms, the optimal search rule may imply that consumers first continue searching another firm, and then go back to a previously sampled firm before all firms are sampled. Section 7 concludes with a discussion, while proofs are given in two Appendices.

\section{The model and equilibrium concept}

The sequential search model we analyze is based on the homogeneous goods models studied in Dana (1994) and Janssen et al. (2011). ${ }^{7}$ The basic model we analyze in the next three Sections

\footnotetext{
5 A similar conclusion is drawn in a recent paper on insurance markets (see, Honka and Chintagunta, 2017).

6 This last observation can also be rationalized by assuming that consumers face an increasing search cost.

7 Search models for heterogeneous goods typically follow the model developed by Wolinsky (1986). In that model, firms choose pure price strategies as uncertainty resulting from the match distribution of product variety is exogenously
} 
incorporates cost uncertainty in a duopoly version of Stahl (1989). ${ }^{8}$ Firms produce a homogeneous good and compete in prices. The production cost of each firm equals $c$, which can take one of two values, $c \in\left\{c_{L}, c_{H}\right\}, c_{L} \leq c_{H}$. Production cost is common for both firms. Denote by $\alpha$ the probability that $c=c_{H}$, where $0<\alpha<1$. Firms observe their production cost, but consumers do not. After observing the realization of cost, firms simultaneously set prices. We denote the (symmetric), perhaps degenerate, price distributions chosen by firms by $F_{L}(p)$ and $F_{H}(p)$ when cost is low or high, respectively. The highest price which will be charged by low and high cost firms is denoted by $\bar{p}_{L}$ and $\bar{p}_{H}$, respectively, whereas the respective lowest prices will be denoted by $\underline{p}_{L}$ and $\underline{p}_{H}$. Each firm's objective is to maximize profits, taking the prices charged by the other firm and consumers' search behavior as given.

The demand side of the market is represented by a unit mass of risk-neutral consumers with identical preferences and unit demand. A fraction $\lambda \in(0,1)$ of consumers, shoppers, have a zero search cost. These consumers sample all prices and buy at the lowest price. The remaining fraction of $1-\lambda$ consumers - non-shoppers - search sequentially and have a positive search cost $s>0$ to obtain one additional price quote. They visit each of the two firms at their first search with equal probability. These consumers face a non-trivial problem when searching for low prices, as they have to trade off the search cost with the expected benefit from search. After observing their first price quote, non-shoppers update their beliefs about firms' underlying production costs using Bayes' rule. Consumers can always go back to previously visited firms, incurring no additional cost. ${ }^{9}$ We denote the probability that non-shoppers buy after observing price $p$ by $\beta(p)$. With the remaining probability $1-\beta(p)$ these consumers continue to search. As consumers do not know the underlying production cost, $\beta(p)$ does not depend on the cost realization. Denote by $\rho_{i}$ the consumers' reservation price if they were to infer that the firms' production cost equals $c_{i}$ for sure, $i=L, H$.

The timing of the model is as follows. First, Nature chooses $c$ for both firms. After observing $c$, firms simultaneously decide on their prices. Finally, consumers search and make their purchase decisions.

We consider Perfect Bayesian Equilibria of the game. A (symmetric) equilibrium is a tuple of $(i)$ pricing strategies $F_{i}(p), i=L, H$ such that any $p$ in the support of $F_{i}$ maximizes firm $i$ 's profit, (ii) an optimal search strategy $\beta(p)$ minimizing the expected price (including search cost) at which a consumer buys given her beliefs, and (iii) beliefs that are consistent with firms' pricing strategies on the equilibrium path.

It is by now a standard argument in the search literature with symmetric information that due to the presence of shoppers and non-shoppers there do not exist equilibria with mass points in the price distributions. This argument continues to hold in our model with asymmetric information as far as pure pricing strategies are concerned: even if all non-shoppers continue to search, an undercutting firm will sell to all shoppers and non-shoppers that first visit that firm. In the present model, this argument does not extend, however, to ruling out pricing distribution with mass

\footnotetext{
imposed. Janssen and Shelegia (2015b) introduce common cost uncertainty in that model and show that firms continue to choose pure strategies and consumers learning the state of the world upon observing a price follow reservation price strategies. Thus, the issues we point at in this paper with the non-existence of RPE are not relevant in that alternative search context.

8 It is not difficult to extend the analysis to oligopoly situations if we replace sequential search with newspaper search, in which upon paying a search cost (after observing the first search) consumers are immediately informed about all $(N-1)$ other prices. Extending the analysis with sequential search to oligopoly markets is not straightforward and we discuss the extent to which we can generalize the results in Section 6.2.

9 Janssen and Parakhonyak (2014) analyze the case where this assumption is replaced by costly recall.
} 
points. Given that equilibria have to be in mixed strategies and that we prove that equilibria without mass points always exist, we restrict our attention to mixed pricing strategies without atoms.

As explained in the Introduction, the existing literature focuses on reservation price equilibria, which are defined as follows.

Definition 1. A Perfect Bayesian Equilibrium is a Reservation Price Equilibrium if there exists a $p_{0}$ such that $\beta(p)=1$ for all $p \leq p_{0}$ and $\beta(p)=0$ for all $p>p_{0}$.

When investigating non-RPE, we focus on equilibria satisfying the logic of the D1 criterion (Cho and Sobel, 1990). ${ }^{10}$ The D1 criterion was developed in the context of pure signaling games with one sender. Our model is a two-sender game, where the beliefs of the receivers (the nonshoppers) are only based on the single price they have observed. As firms are of the same type, the out-of-equilibrium belief of non-shoppers is simply a mapping from the observed price to the type distribution of cost, as in the one-sender game.

Consider any perfect Bayesian equilibrium where the equilibrium profit of firm $j$ when it is of type $i$ is given by $\pi_{i}^{j *}, i=H, L$. Consider any $p$ outside the support of the equilibrium price distribution. This out-of-equilibrium price generates a set of possible optimal actions of the receiver (non-shopper). Let $B_{j}(p)$ be the set of a firm $j$ 's total demand from shoppers and non-shoppers that can be generated by buying probabilities $\beta_{j}(p)$ of non-shoppers (at firm $j$ at price $p$ ) that are best responses to some non-shoppers' belief. A $q_{j}(p) \in B_{j}(p) \subset[0,1]$ is an element of this set. For out-of-equilibrium price that are larger than the highest price charged along the equilibrium path, shoppers will not buy and demand at such a price is bounded by $\frac{1-\lambda}{2}$. The D1 refinement compares the sets of demands $\left\{q_{j} \in B_{j}(p):\left(p-c_{i}\right) q_{j} \geq \pi_{i}^{j *}\right\}$ for which it is gainful for different types $i$ of firm $j$ to deviate to price $p$. If for $i, i^{\prime} \in\{H, L\}, i^{\prime} \neq i$,

$$
\left\{q_{j} \in B_{j}(p):\left(p-c_{i}\right) q_{j} \geq \pi_{i}^{j *}\right\} \subset\left\{q_{j} \in B_{j}(p):\left(p-c_{i^{\prime}}\right) q_{j}>\pi_{i^{\prime}}^{j *}\right\}
$$

where " $\subset$ " stands for strict inclusion, the D1 logic requires that the out-of-equilibrium beliefs of non-shoppers (upon observing a unilateral deviation by firm $j$ to price $p$ ) should assign zero probability to the event that firm $j$ is of type $i$ and thus (as there are only two types and firms have a common type), assign probability one to firm $j$ being of type $i^{\prime} .^{11}$ Intuitively, as type $i^{\prime}$ has an incentive to deviate to $p$ for a larger set of responses by the non-shoppers than type $i$, the first type is said to have a stronger incentive to deviate.

In addition, we will focus on equilibria where $\beta(p)$ is continuously differentiable almost everywhere as follows. Let $P=[0, \bar{p}]$, with $\bar{p}=\max \left\{\bar{p}_{L}, \bar{p}_{H}\right\}$ and define sets $P_{(0,1)}=\{p: p \in$ $P, 0<\beta(p)<1\}, P_{1}=\{p: p \in P, \beta(p)=1\}$ and $P_{0}=\{p: p \in P, \beta(p)=0\} .{ }^{12}$ We consider

\footnotetext{
10 Ideally, we want to make sure that the upper bound of the price distribution does not depend on arbitrary out-ofequilibrium beliefs. In the next section we show that this can be achieved when consumers, after observing the highest price charged in equilibrium, believe that they are in a high cost environment. This implies that independent of their beliefs at higher prices consumers prefer to search for a better price after observing such higher price. As we show later the D1 criterion implies that after observing a price equal to the upper bound of the price distribution consumers believe that they are in a high cost environment. Accordingly, if a pricing strategy profile is part of an equilibrium for beliefs that satisfy the D1 criterion, it is also part of an equilibrium for any other out-of-equilibrium beliefs.

11 A similar treatment is given in Janssen and Roy (2010) for a more complicated inference problem where consumers observe all prices and there are $N$ firms.

12 Note that it is not the case that all sets $P_{i}$ that satisfy the criteria are necessarily convex. In Section 4 we provide examples of equilibria where the set of all prices with $\beta=1$ or with $\beta \in(0,1)$ are non-convex.
} 
equilibria, where $\beta(p)$ is continuously differentiable in the interior of these sets, and refer to this as $\beta(p) \in C^{1}$. $^{13}$

Definition 2. A symmetric Perfect Bayesian Equilibrium is a non-reservation price equilibria (non-RPE) that satisfies the D1 logic and is sufficiently smooth if (i) it does not satisfy Definition 1 , (ii) $\beta(p) \in C^{1}$, (iii) $F_{i}(\cdot)$ are continuous and (iv) consumers' out-of-equilibrium beliefs are consistent with the D1 logic.

Thus, from the set of equilibria that do not satisfy Definition 1, we focus on those equilibria that satisfy the D1 logic and that are sufficiently smooth. For easy reference, we refer to such equilibria in the rest of the paper as non-reservation price equilibria (non-RPE). There may exist other equilibria that do not satisfy the properties of a RPE and that do not satisfy the D1 requirement and are not smooth. We do not consider these equilibria in our paper as we show that even with the additional requirements of D1 and smoothness, we can guarantee existence. Moreover, the equilibria we consider are interesting in their own right and do not depend on arbitrary out-of-equilibrium beliefs or on more technical issues related to non-smoothness.

\section{Reservation price equilibria}

In this Section, we summarize some existing results on RPE, (i) prove that they do not exist if the cost uncertainty is large and (ii) prove that they do not satisfy the D1 logic even if they do exist.

Dana (1994) has characterized RPE in our model, while Janssen et al. (2011) have generalized that analysis to $N$ firms and production cost being distributed according to a continuous distribution function. For $N=2$, Janssen et al. (2011) showed that the equilibrium price distribution for cost realization $c_{i}$ is given by

$$
F\left(p \mid c_{i}\right)=1-\frac{1-\lambda}{2 \lambda} \frac{\bar{p}-p}{p-c_{i}}, \quad i=L, H
$$

with support on $\left[\underline{p}_{i}, \bar{p}\right]$ with $\underline{p}_{i}=\frac{2 \lambda}{1+\lambda} c_{i}+\frac{1-\lambda}{1+\lambda} \bar{p}, i=L, H$, and $\bar{p}=\rho$, the consumers' reservation price. The derivation of the mixed strategy distribution follows from the fact that given a firm's own price $p$, its profit is given by

$$
\left[\lambda\left(1-F_{i}(p)\right)+\frac{1-\lambda}{2}\right]\left(p-c_{i}\right), \quad i=L, H,
$$

and that in a mixed strategy equilibrium, this profit has to be equal to the profit the firm gets if it sets a price equal to the upper bound $\rho$ of the price distribution, $\frac{1-\lambda}{2}\left(\rho-c_{i}\right)$. It follows that the respective density functions are given by ${ }^{14}$

$$
f_{i}(p)=\frac{1-\lambda}{2 \lambda} \frac{\rho-c_{i}}{\left(p-c_{i}\right)^{2}}, \quad i=L, H,
$$

and that the reservation price $\rho$ is implicitly determined by

\footnotetext{
$\overline{13}$ Smoothness of the $\beta(p)$ function is needed to assure that the process of Bayesian updating of the underlying cost is continuous on the relevant sets.

14 As $p \geq \underline{p}_{i}>c_{i}$ it follows that the densities are finite.
} 


$$
\rho=s+\frac{\alpha f_{H}(\rho)}{\alpha f_{H}(\rho)+(1-\alpha) f_{L}(\rho)} E\left(p \mid c_{H}\right)+\frac{(1-\alpha) f_{L}(\rho)}{\alpha f_{H}(\rho)+(1-\alpha) f_{L}(\rho)} E\left(p \mid c_{L}\right) .
$$

The latter equation basically says that at the reservation price, the consumer must be indifferent between buying now (and paying a price $\rho$ ) and continuing to search, which costs $s$, and paying the expected price, giving the consumer updates her beliefs about whether the underlying cost is high or low. Janssen et al. (2011) show that the expression for the reservation price can be rewritten as $\rho=E(c \mid \rho)+\frac{s}{1-\gamma}$, where $\gamma$ is a parameter that only depends on $\lambda$ and $N$ (which equals two in our case).

A few results that will be used later follow from this characterization. First, no firm charges prices above the reservation price. Second, the low and high cost densities at the reservation price are positive, i.e., $f_{i}(\rho)>0$. This implies that the posterior belief that cost is low after observing the reservation price, $\operatorname{Pr}\left(c_{L} \mid \rho\right)$, which is given by

$$
\operatorname{Pr}\left(c_{L} \mid \rho\right)=\frac{(1-\alpha) f_{L}(\rho)}{\alpha f_{H}(\rho)+(1-\alpha) f_{L}(\rho)},
$$

is strictly larger than 0 (and strictly smaller than 1). Third, for any price $p$ that is in the support of the equilibrium price distributions in both states of the world, the density in the low cost state is smaller than the density in the high cost state. This implies that $F_{H}(p)$ first-order stochastically dominates $F_{L}(p)$. Thus, the expected price when cost is low is smaller than the expected cost when cost is high, i.e.,

$$
E\left(p \mid c_{L}\right)<E\left(p \mid c_{H}\right) .
$$

Formally, Dana (1994) and Janssen et al. (2011) only show that a sufficient condition for the existence of an RPE is that the cost uncertainty $c_{H}-c_{L}$ is sufficiently small and that the search cost $s$ is sufficiently large. To show that these are also necessary conditions, we first show that if $c_{H}-c_{L}$ is sufficiently large or $s$ is sufficiently small an RPE does not exist irrespective of the out-of-equilibrium beliefs.

\section{Proposition 1. If $c_{H}-c_{L}$ is sufficiently large, or $s$ is sufficiently small an RPE does not exist.}

We illustrate the " $c_{H}-c_{L}$ being sufficiently large" part of Proposition 1 in Fig. 1, where we depict the solutions to equations (1)-(3) for various cost differences. If an RPE exists, it should be the solution to equations (1)-(3). As the Figure shows, consumers' beliefs discretely change at $\underline{p}_{H}$, as for all $p<\underline{p}_{H}$ consumers infer that they are in a low cost environment. When $c_{L}=30$, as on the left pane of the Figure, this does not cause a problem with the equilibrium construction, as the net benefit of search is still negative. However, if $c_{L}=8$, the reservation price $\rho_{L}$ conditional on being in the low cost environment is smaller than $\underline{p}_{H}$, and for prices just below $\underline{p}_{H}$ the net benefit of search is positive. Therefore, an RPE does not exist as non-shoppers prefer to continue to search for lower prices after observing a price just below $\underline{p}_{H} \cdot{ }^{15}$

The result of Proposition 1 also holds for heterogeneous search costs. In order to see this, fix some (sufficiently large) $c_{H}$ and (sufficiently small) $s$. Then, as it follows from the proof

\footnotetext{
15 Note that the non-existence of an RPE for large cost uncertainty (and the "gap in the set of accepted prices") does not depend on the assumption of a binary cost state. If we think of $c_{H}$ and $c_{L}$ as the upper and lower bounds of the set of possible cost states with a distribution that is concentrated around these upper and lower bounds, then it continues to be true that the incentive to continue searching may change dramatically (although not discontinuously) when a price just below or just above $\underline{p}_{H}$ is observed. In fact, Janssen et al. (2011) contains an example where an RPE fails to exist if cost is uniformly distributed.
} 
$c_{L}=30, c_{H}=40, \lambda=0.1, \alpha=0.1, \mathrm{~s}=2$

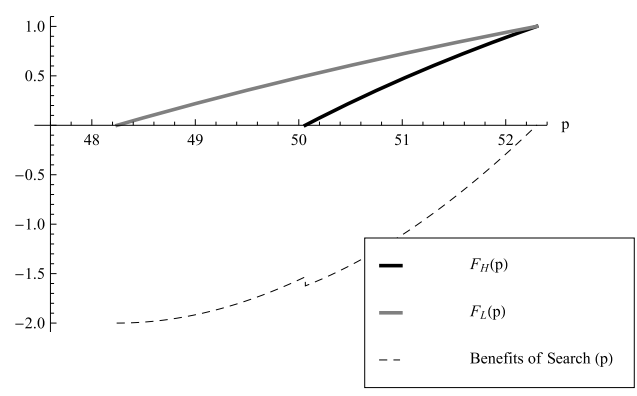

(a)
$c_{L}=8, c_{H}=40, \lambda=0.1, \alpha=0.1, \mathrm{~s}=2$

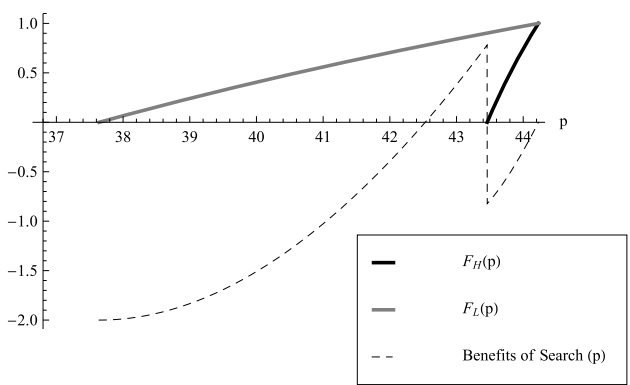

(b)

Fig. 1. RPE and incentives to search.

of Proposition 1 there is a sufficiently small $c_{L}$ such that the reservation price, conditional on knowing that the cost is low, is below $c_{H}$. As a high-cost firm will not price below $c_{H}$, this implies that consumers would search for prices just below $c_{H}$. Now, if search costs are distributed on some interval $\left[s^{\prime}, s\right]$ below the search costs considered in Proposition 1, the distribution of prices for low-cost firms cannot be higher than the one for a single search cost $s$, and therefore consumers would still search actively for prices just below $c_{H}$.

We next show that if an RPE does not exist, any equilibrium without mass points should have a region of prices where non-shoppers actively search (search with positive probability).

Proposition 2. For any search cost $s>0$, if $c_{H}-c_{L}$ is sufficiently large, then consumers search with positive probability in any PBE with continuous $F_{i}(\cdot)$.

Basically, from the first part of the proof of Proposition 1 we can use the fact that $\rho_{L}<\underline{p}_{H}$ for $c_{H}-c_{L}$ being sufficiently large. This implies there should be a region of prices just above $\underline{p}_{H}$ where there is active search to keep the low cost firm indifferent between charging these prices and $\rho_{L}$. The cut-off value of $c_{H}-c_{L}$ where an RPE does not exist anymore in Proposition 1 is be exactly the cut-off value where consumers search with positive probability in Proposition 2.

Finally, we show that any RPE, even if it exists for some out-of-equilibrium beliefs, does not satisfy the D1 logic. The D1 logic asks which type of firm (high or low cost) has most incentive to deviate to prices above the reservation price. It turns out that high cost firms have stronger incentives to deviate; see the proof of the next Proposition for details. This implies that if a consumer continues to search after observing an out-of-equilibrium price $\rho+\varepsilon$, for small $\varepsilon$, she expects to pay $E\left(p \mid c_{H}\right)+s$, including the search cost. If the consumer buys immediately, she pays $\rho+\varepsilon$, which using (7) can be rewritten as

$$
\varepsilon+\operatorname{Pr}\left(c_{H} \mid \rho\right) E\left(p \mid c_{H}\right)+\operatorname{Pr}\left(c_{L} \mid \rho\right) E\left(p \mid c_{L}\right)+s .
$$

This expression is strictly smaller than the expected payment in case of search if, and only if,

$$
\operatorname{Pr}\left(c_{L} \mid \rho\right)\left(E\left(p \mid c_{H}\right)-E\left(p \mid c_{L}\right)\right)>\varepsilon \text {. }
$$

From (2) and (4) it follows that the LHS is strictly positive. Thus, one can choose $\varepsilon$ sufficiently small so that at $\rho+\varepsilon$ it is optimal to buy. Firms would then, however, have an incentive to deviate and set these higher prices defying the notion of equilibrium. The next Proposition formalizes this logic and extends it to all equilibria without active search. 
Proposition 3. All perfect Bayesian equilibria in which non-shoppers buy with probability one in the first search round and in which $F_{i}(p)$ is continuous, do not satisfy the D1 logic.

There are two important corollaries, which follow immediately from Proposition 3. First, as in any RPE firms' pricing distributions are atomless (see, e.g. Stahl, 1989), and there is no active search (see Dana, 1994), we immediately have the following corollary.

Corollary 1. All reservation price equilibria do not satisfy the D1 logic.

Second, by Definition 2 we have

Corollary 2. In any non-reservation price equilibrium non-shoppers search with positive probability.

Note that the D1 logic may be extreme in the sense that it requires that $\operatorname{Pr}\left(c_{L} \mid p\right)=0$ for any $p>\rho$. Following the logic of the proof, even weaker restrictions on the out-of-equilibrium beliefs are sufficient to eliminate RPE, however. RPE requires that after observing prices above the reservation price, consumers infer that cost is low with sufficiently high probability. Thus, RPE do not exist either if the out-of-equilibrium beliefs are discontinuous and such that there exists a $k>0$ such that $\operatorname{Pr}\left(c_{L} \mid p\right)+k<\operatorname{Pr}\left(c_{L} \mid \rho\right)$ for any $p>\rho$. RPE can, however, be compatible with out-of-equilibrium beliefs $\operatorname{Pr}\left(c_{L} \mid p\right)$ that are strictly decreasing for $p>\rho$ and that are continuous at $\rho$.

\section{Characterization and existence of non-RPE}

In any non-RPE, a firm's profit $\pi\left(p \mid c_{i}\right)$ when setting price $p$ and $\operatorname{cost}$ is $c_{i}, i=H, L$ can be written as

$$
\begin{aligned}
& \pi\left(p \mid c_{i}\right)=\left[\lambda\left(1-F_{i}(p)\right)+\frac{1-\lambda}{2} \beta(p)+\frac{1-\lambda}{2}(1-\beta(p))\left(1-F_{i}(p)\right)+\right. \\
& \left.\frac{1-\lambda}{2} \int_{p}^{\bar{p}}(1-\beta(\widetilde{p})) f_{i}(\tilde{p}) d \widetilde{p}\right]\left(p-c_{i}\right) .
\end{aligned}
$$

This expression can be understood as follows. First, a firm only attracts shoppers if the other firm charges a higher price, which occurs with probability $1-F_{i}(p)$. The number of non-shoppers buying from firm $i$ gives a more complicated expression. There is a fraction $(1-\lambda) / 2$ of nonshoppers that randomly first visits firm $i$, and buys immediately from that firm with probability $\beta(p)$. The remaining non-shoppers that randomly first visit firm $i$ continue searching the other firm and come back to firm $i$ if the other firm has a higher price. Finally, the non-shoppers that first visit the other firm and decide to continue to search buy from firm $i$ if it has a lower price. As firm $i$ does not know which price the other firm charges, this expression involves an expected number of consumers.

To characterize the price distributions of non-RPE, we first show that the upper bounds of the low and high cost price distributions have to be identical. If this were not the case, there would be a region of prices above the upper bound of, say, the low cost distribution that are only chosen 
by high cost firms, and this would imply that $\beta(p)=1$. Low cost firms would then have an incentive, however, to deviate to such prices.

Lemma 1. In PBE with continuous $F_{i}(p)$, thus, in any non-RPE, $\bar{p}_{L}=\bar{p}_{H} \equiv \bar{p}$.

Without mass points, a firm setting a price equal to the upper bound $\bar{p}$ of the price distribution will not sell to the shoppers and their profits will be equal to $\frac{1-\lambda}{2} \beta(\bar{p})\left(\bar{p}-c_{i}\right)$. As in equilibrium, for any price in the support of the price distribution this expression has to be equal to (5), we have that

$$
\begin{aligned}
\lambda\left(1-F_{i}(p)\right)+\frac{1-\lambda}{2} \beta(p)+ & \frac{1-\lambda}{2}(1-\beta(p))\left(1-F_{i}(p)\right)+ \\
& \frac{1-\lambda}{2} \int_{p}^{\bar{p}}(1-\beta(\tilde{p})) f_{i}(\tilde{p}) d \tilde{p}=\frac{1-\lambda}{2} \beta(\bar{p}) \frac{\bar{p}-c_{i}}{p-c_{i}} .
\end{aligned}
$$

At intervals of prices in the support of the price distribution where $\beta(p)=1$, or, $\beta(p)=$ 0 , this equation can be solved for $F_{i}(p)$ in a straightforward manner. As we concentrate on equilibria where $\beta(p)$ is continuously differentiable in the interior of $P_{(0,1)}$, equation (6) can be transformed into an exact differential equation that can be solved as shown in the proof of the following Proposition.

Proposition 4. If $F(p)$ is a price distribution in a non-RPE, then it should be of the following form:

$$
F_{i}(p)= \begin{cases}\frac{2 \sqrt{1-(1-\lambda) \beta(\bar{p})}-\int_{p}^{\bar{p}} \frac{(1-\lambda) \beta(\bar{p})\left(\bar{p}-c_{i}\right)}{\left(\widetilde{p}-c_{i}\right)^{2} \sqrt{1-(1-\lambda) \beta(\widetilde{p})}} d \tilde{p}}{2 \sqrt{1-(1-\lambda) \beta(p)}} & \text { if } p \in P_{(0,1)} \\ 1-\frac{1-\lambda}{2 \lambda}\left[\beta(\bar{p}) \frac{\bar{p}-c_{i}}{p-c_{i}}-1-\int_{p}^{\bar{p}}(1-\beta(\widetilde{p})) f_{i}(\widetilde{p}) d \widetilde{p}\right] & \text { if } p \in P_{1} \\ 1-\frac{1-\lambda}{1+\lambda}\left[\beta(\bar{p}) \frac{\bar{p}-c_{i}}{p-c_{i}}-\int_{p}^{\bar{p}}(1-\beta(\widetilde{p})) f_{i}(\widetilde{p}) d \tilde{p}\right] & \text { if } p \in P_{0}\end{cases}
$$

Using the characterization of the price distributions, we can now state that $F_{H}(p)$ first-order stochastically dominates the low cost distribution $F_{L}(p)$. Thus, as in RPE, we continue to have the expected price when cost is low, $E\left(p \mid c_{L}\right)$, being lower than the expected price when cost is high, $E\left(p \mid c_{H}\right)$.

Corollary 3. In any non-RPE, for all $p<\bar{p}, F_{L}(p) \geq F_{H}(p)$ and whenever $0<F_{H}(p)<1$, $F_{L}(p)>F_{H}(p)$.

Using these characterizations of the distribution functions, it is not too difficult to see that if we want that the upper bound of the distributions $\bar{p}$ is not determined by arbitrary out-of-equilibrium beliefs, it must be the case that after observing $\bar{p}$ consumers believe that firms have high cost for sure, and that given this inference, non-shoppers are indifferent between buying now and continuing to search. If this were not the case, and non-shoppers had out-of-equilibrium beliefs such that $\operatorname{Pr}\left(c=c_{H} \mid p\right)=1$ for prices $p>\bar{p}$, then they would prefer to buy at these prices, giving firms an incentive to deviate (see the proof of Proposition 3 for details). Thus, the density of the low cost distribution at the upper bound must be equal to zero, 


$$
f_{L}(\bar{p})=0
$$

and the upper bound of the price distributions has to be equal to the reservation price in the case where consumers know cost is high, i.e.,

$$
\int_{\underline{p}_{H}}^{\bar{p}} F_{H}(p) d p=s
$$

As $F_{H}(p)$ first-order stochastically dominates $F_{L}(p)$ this implies that if an out-of-equilibrium price larger than $\bar{p}$ is observed, consumers will always want to continue to search independent of their beliefs of the underlying cost.

To fully characterize an equilibrium of the model, we have to inquire into the non-shoppers' equilibrium strategy, $\beta(p)$, with $0 \leq \beta(p) \leq 1$. Optimal search behavior implies that whenever $0<\beta(p)<1$ the non-shopper is indifferent between buying now and continuing to search, implying that

$$
\frac{(1-\alpha) f_{L}(p)}{(1-\alpha) f_{L}(p)+\alpha f_{H}(p)} \Phi_{L}(p)+\frac{\alpha f_{H}(p)}{(1-\alpha) f_{L}(p)+\alpha f_{H}(p)} \Phi_{H}(p)=s,
$$

where $\Phi_{i}(p)=\int_{0}^{p} F_{i}(x) d x$. This equation says that after a non-shopper observes price $p$ she will update her beliefs about the underlying cost of the firms and given these updated beliefs concludes that buying now yields the same expected pay-off as continuing to search. Optimal search behavior also implies that the non-shoppers strictly prefer to buy $(\beta(p)=1)$ if the LHS of (10) is strictly smaller than $s$ and that the non-shoppers strictly prefer to search $(\beta(p)=0)$ if the LHS of (10) is strictly larger than $s$. Together with (7) this behavior characterizes an equilibrium.

As shown in Appendix A, equation (10) defines a differential equation which, starting from initial conditions for $\bar{p}$ and $\beta(\bar{p})$, defines the function $\beta(p)$ going downward. ${ }^{16}$ This function can continue to satisfy $0<\beta(p)<1$ or it may at some price point $p$ reach the boundaries $\beta(p)=1$ or $\beta(p)=0$. If for some prices $\beta(p)=1$, the following Lemma shows that (10) implies that in any equilibrium $\beta^{\prime}(p)=0$ has to hold at the largest price point $p$ where $\beta(p)=1$.

Lemma 2. Let $p^{*}$ be such that $\beta\left(p^{*}\right)=1$ and for any sufficiently small $\epsilon>0 \beta\left(p^{*}+\epsilon\right)<1$. Suppose that $p^{*}$ is in the interior of the support of $F_{i}(p), i=L, H$. Then in equilibrium it must be that $\beta^{\prime}\left(p^{*}\right)=0$.

We will now inquire into the existence question. The main question is whether for all parameter values $c_{L}, c_{H}, \lambda, \alpha$ and $s$ we can find values of $\bar{p}$ and $\beta(\bar{p})$ such that equation (7) defines proper distribution functions that are upward sloping, and that the search strategy of non-shoppers satisfies the optimality condition (10). ${ }^{17}$ Our main Theorem shows that a non-reservation price equilibrium as defined in Definition 2 exists for all values of the exogenous parameters.

Theorem 1. For any values of $s, \lambda, c_{L}, c_{H}$ and $\alpha$ a non-reservation price equilibrium as defined in Definition 2 exists. The equilibrium price distributions are characterized by (7), while non-shopper's behavior is determined by (10) whenever $0<\beta(p)<1$.

\footnotetext{
16 Note that (10) implies that we should have $\beta^{\prime}(\bar{p})=-\beta(\bar{p}) /\left(\bar{p}-c_{L}\right)$ as derived in Proposition A.1 in Appendix A.

17 In Appendix B we show that for given $F_{L}(p)$ and $F_{H}(p)(10)$ essentially is a differential equation that determines the function $\beta(p)$ up to the boundary condition $\beta(\bar{p})$.
} 


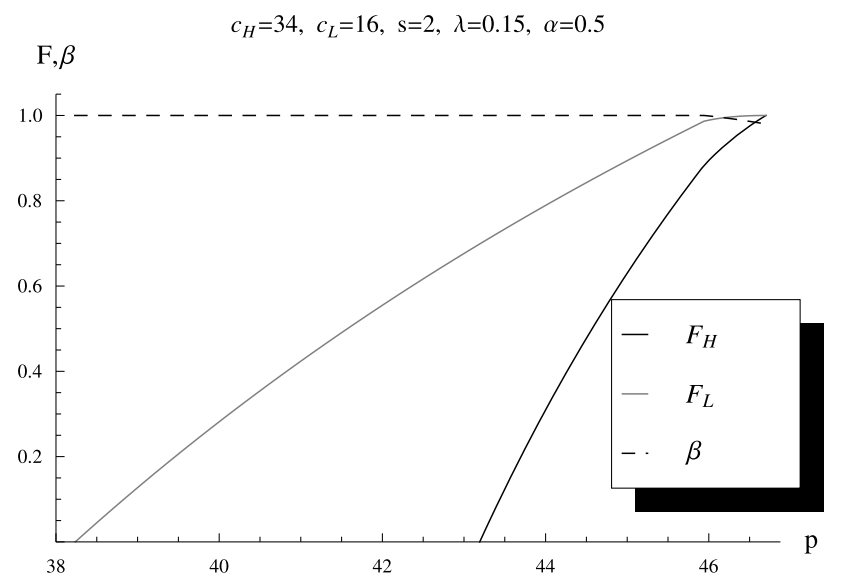

Fig. 2. No-gap equilibrium.

The proof is constructive and consists of several Lemmas. It is formally developed in Appendix B. For a range of parameter values the equilibrium is not unique, while for other parameter values it is unique. We now extensively describe how for any set of parameter values we construct an equilibrium.

The four-step procedure works as follows. First, we show that we can always find values $\bar{p}$ and $\beta(\bar{p})$ such that the solution of the system of equations (7) and (10) satisfies two boundary conditions: $\beta^{\prime}\left(p^{*}\right)=0$ and $\int_{\underline{p}_{H}}^{\bar{p}} F_{H}(p) d p=s$, where $p^{*}$ is defined in Lemma 2. Given the values of $\bar{p}$ and $\beta(\bar{p})$, we take $\beta(p)=1$ for all $p<p^{*}$ and define $\rho_{L}$ such that

$$
\int_{\underline{p}_{L}}^{\rho_{L}} F_{L}(p) d p=s .
$$

Here we can distinguish between two cases. If $\rho_{L} \geq \underline{p}_{H}$ then we claim we have found a nonreservation price equilibrium without a gap in both price distributions. In this so-called no-gap equilibrium, as in any other non-RPE, conditions (8) and (9) must be satisfied, so after observing $\bar{p}$ non-shoppers are indifferent between buying now and continuing to search. At all prices $p$ with $p^{*}<p<\bar{p}$ both $f_{L}(p)$ and $f_{H}(p)$ are strictly positive and (10) guarantees that non-shoppers are indifferent over the whole interval. Finally, Lemma A.3 in Appendix B shows that if we specify $\beta(p)=1$ for all $p<p^{*}$, then the equilibrium density functions are such that consumers indeed prefer to buy at all these prices. Lemmas A.1 and A.2 prove that (7) always define proper price distributions. An example of a no-gap equilibrium is given in Fig. 2. This Figure illustrates that at high prices $\beta(p)<1$ and at lower prices $\beta(p)=1$ and the price distributions do not have a gap. Fig. 2 also illustrates that the demand of individual consumers is downward sloping.

The second possibility is that the condition $\rho_{L} \geq \underline{p}_{H}$ is violated. In that case, it is natural to have out-of-equilibrium beliefs satisfying $\operatorname{Pr}\left(c_{L} \mid p\right)=1$ for all $p \in\left(\rho_{L}, \underline{p}_{H}\right)$ implying that consumers would not like to buy at these prices and $\beta(p)=0$. This out-of-equilibrium belief not only follows from the D1 logic, but also from the weaker notion of the Intuitive Criterion (Cho 
and Kreps, 1987). ${ }^{18}$ For given values of the other parameters, $\rho_{L}<\underline{p}_{H}$ will arise when the cost difference $c_{H}-c_{L}$ is sufficiently large, i.e., the same reason why an RPE may fail to exist for any out-of-equilibrium belief (see Proposition 1 in Section 3).

Below, in steps (2)-(4) we indicate different ways an equilibrium with a gap can be constructed. A gap equilibrium configuration presumes that $\beta(p)=0$ for all $p \in\left(\rho_{L}, \underline{p}_{H}\right)$ and $F_{L}\left(\rho_{L}\right)=F_{L}\left(\underline{p}^{\prime}\right)$, where $\rho_{L}<\underline{p}^{\prime} \leq \underline{p}_{H}$. In steps (2)-(3) we claim that $\underline{p}^{\prime}=\underline{p}_{H}$ as $\beta\left(\underline{p}_{H}\right)>0$ requires that for any price $p \in\left(\rho_{L}, \underline{p}_{H}\right)$ the low cost density function is equal to 0 . Moreover, for all $p \in\left[\underline{p}_{L}, \rho_{L}\right)$ we must have $\beta(p)=1$ due to the fact that at $\rho_{L}$ non-shoppers are indifferent between buying and not buying even if they know that the underlying cost is low. Finally, it must be the case that $\beta\left(\underline{p}_{H}\right)<1$ as otherwise the low quality firms cannot be indifferent between setting $\underline{p}_{H}$ and $\rho_{L}$ as the chance to attract shoppers is the same at both prices.

The second step in the equilibrium construction procedure is then to try to construct a nonreservation price equilibrium (in case there is no "no-gap equilibrium") that is close to the "no-gap equilibrium" in that there also exists an interval of prices $p \in\left[x, p^{*}\right]$ with $\underline{p}_{H}<x<p^{*}$ where $\beta(p)=1$. The main difference with the "no-gap equilibrium" is that $\beta(p)<1$ for prices $p$ with $\underline{p}_{H}<p<x$. In addition to the two parameter values and two boundary conditions we encountered above in the "no-gap equilibrium", we have one more parameter value that we can choose, namely $x$ to make sure that $\pi_{L}\left(\rho_{L}\right)=\pi\left(\underline{p}_{H}\right)$, where $\rho_{L}$ has to satisfy (11). In the proof of the main theorem in Appendix B, we show that if there is no "no-gap equilibrium" and $\rho_{L}<\underline{p}_{H}$ we can satisfy this third boundary condition as well with a positive size of a gap, provided that we can find an $x$ such that $x \leq p^{*}$. For lack of a better term, we call such a nonreservation price equilibrium, a monopolistic gap equilibrium for the fact that there is a interval of prices $p \in\left[x, p^{*}\right]$ where $\beta(p)=1$ and firms have some "monopoly power" over non-shoppers as they always buy.

Fig. 3 illustrates a monopolistic gap equilibrium. At prices close to $\bar{p}$, but also at prices close to $\underline{p}_{H}$ non-shoppers are indifferent between buying and continuing to search and $\beta(p)<1$. At prices at and close to $\underline{p}_{H}, \beta(p)>0$ and $\beta^{\prime}(p)>0$ and the low cost distribution function is much steeper in this price region than the high cost distribution function. There is a relatively small gap in the low cost price distribution and $\beta(p)=1$ for all $p \leq \rho_{L}$. At the lowest price $p$ such that $\beta(p)=1, \beta(p)$ is not continuously differentiable. ${ }^{19}$

The third step in the procedure starts from the fact that if a no-gap equilibrium does not exist, then a monopolistic gap equilibrium may also fail to exist if we cannot find a value $x \leq p^{*}$ such that all necessary boundary conditions are satisfied. In this case, we try to construct an equilibrium with $0<\beta(p)<1$ for all $p \geq \underline{p}_{H}$. As there is no price $p \geq \underline{p}_{H}$ where $\beta(p)=1$ the condition that $\beta^{\prime}\left(p^{*}\right)=0$ is no longer relevant. So we need the two parameter values $\beta(\bar{p})$ and $\bar{p}$ to satisfy the two boundary conditions (9) and (11) which are relevant for this type of equilibrium, and $\rho_{L}$ is determined from the indifference condition $\pi_{L}\left(\rho_{L}\right)=\pi_{L}\left(\underline{p}_{H}\right)$. Here we

\footnotetext{
18 Intuitively, the reason is as follows: by setting a price equal to $\underline{p}_{H}$ a high cost firm already attracts all shoppers and all non-shoppers that first visited that firm. Of the remaining non-shoppers it will sell to all who continue to search after having visited the first firm. By deviating to a lower price, a firm can never get a higher demand, and lowering the price, can only lower the profits. A low cost firm may have an incentive to deviate to prices $p \in\left(\rho_{L}, \underline{p}_{H}\right)$ if $\beta(p)$ is sufficiently high. As the high cost type does not have an incentive to deviate and the low cost type may have an incentive (depending on the reaction of the non-shoppers), the Intuitive Criterion implies that $\beta(p)=0$ for all $p \in\left(\rho_{L}, \underline{p}_{H}\right)$.

19 Note that Lemma 2 only applies to the largest price $p$ where $\beta(p)=1$.
} 


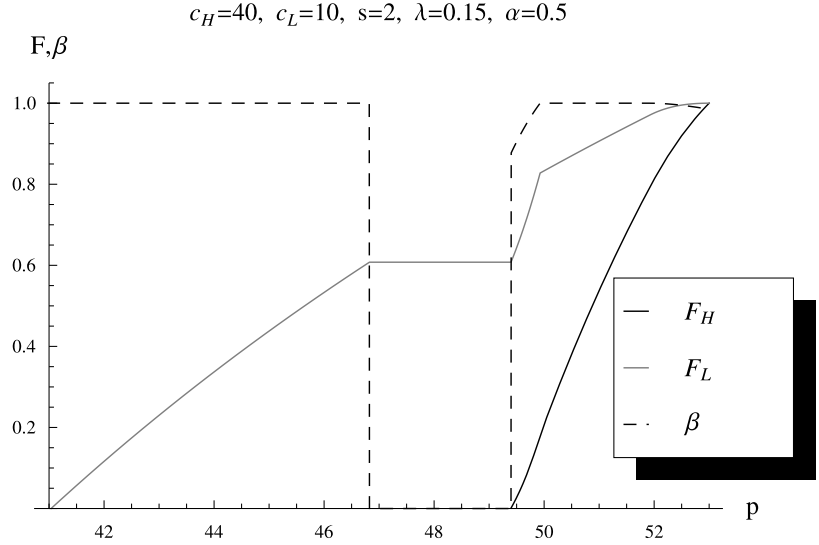

Fig. 3. Monopolistic gap equilibrium.

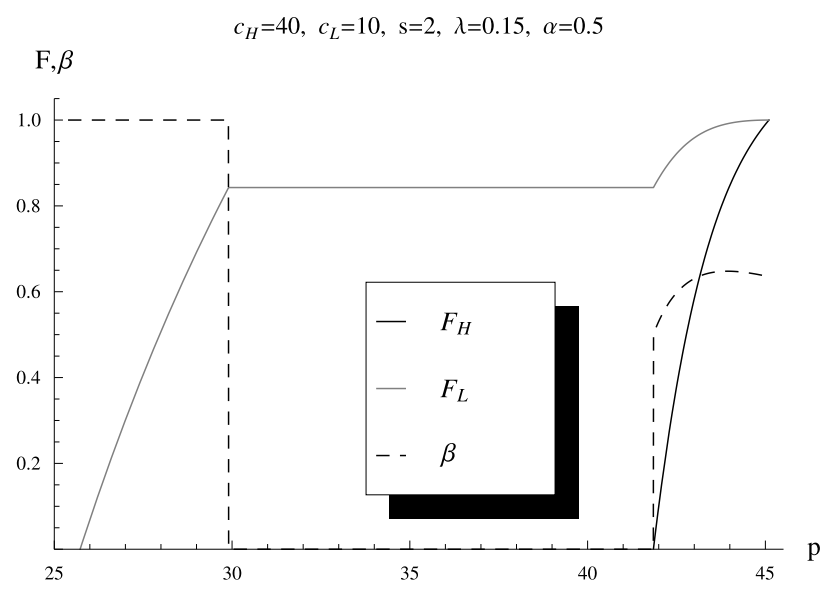

Fig. 4. Regular gap equilibrium.

can get multiple equilibria which can also coexist with a monopolistic gap equilibrium. We call this type of equilibrium where $0<\beta(p)<1$ for all $p \geq \underline{p}_{H}$ a regular gap equilibrium. ${ }^{20}$

Fig. 4 illustrates a regular gap equilibrium. In this equilibrium, there are four regions of prices where non-shoppers exhibit different behavior. At high prices (above $\bar{p}$ ), consumers definitely continue to search. Consumers are indifferent between buying and continuing to search for all prices $p \in\left[\underline{p}_{H}, \bar{p}\right]$ as they update their beliefs about cost being low and the probability of finding lower prices if continuing to search. At prices below $\underline{p}_{H}$ (and above $\rho_{L}$ ) non-shoppers search for sure. Finally, at prices below $\rho_{L}$ non-shoppers buy for sure.

The fourth and final step in the procedure starts from the fact that if neither a no-gap equilibrium nor a monopolistic gap equilibrium exists, the only reason why we cannot find parameter values $\beta(\bar{p})$ and $\bar{p}$ to satisfy the two boundary conditions of a regular gap equilibrium is if the

20 Obviously, an equilibrium where $\beta(p)=1$ at just one point $p \geq \underline{p}_{H}$ is a transition between the monopolistic gap and the regular gap equilibrium. 


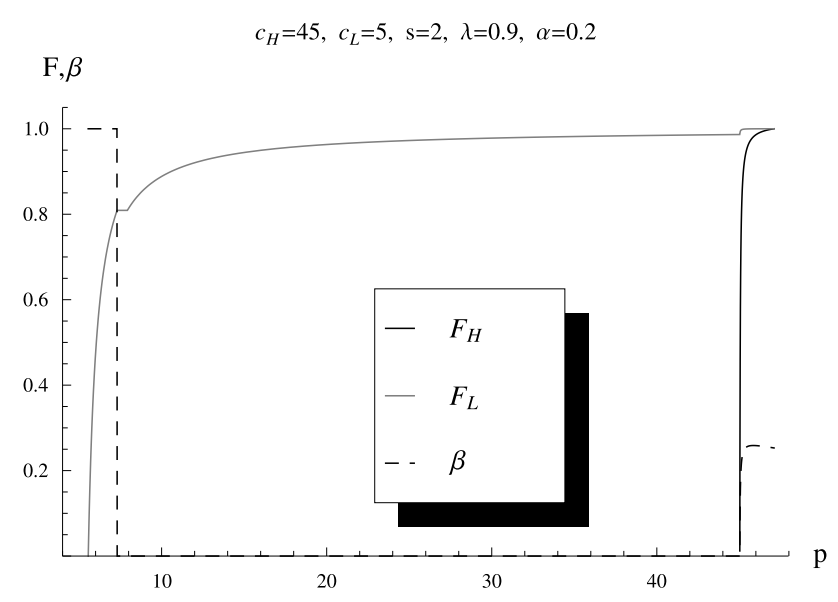

Fig. 5. Competitive gap equilibrium.

constraint $\beta(p)>0$ for all $p \geq \underline{p}_{H}$ cannot be satisfied. From Lemmas A.4-A.7 in Appendix B it follows that if this constraint is violated for some prices $p$, it is certainly violated for all smaller prices. Thus, in our final step we construct an equilibrium where $\beta_{H}\left(\underline{p}_{H}\right)=0$. In this case, low cost firms charge prices with positive probability in the interval $p \in\left[\underline{p}^{\prime}, \underline{p}_{H}\right]$ for some $\rho_{L}<\underline{p}^{\prime}<\underline{p}_{H}$ where $\beta(p)=0$. The third line in equation (7) shows the distribution function of low cost prices in this case. In this case, we need that $F_{L}\left(p^{\prime}\right)=F_{L}\left(\rho_{L}\right)$ and $f_{L}(p)=0$ for all $p \in\left(\rho_{L}, p^{\prime}\right)$ and choose $\beta(\bar{p}), \bar{p}, p^{\prime}$ such that the following three boundary conditions are satisfied: (9), (11) and $\beta\left(\underline{p}_{H}\right)=0$. We call such an equilibrium a competitive gap equilibrium (see Fig. 5) as there is a region of prices that is set by low cost firms where consumers only buy if they know that this is the lowest price in the market. In the last part of the proof of Theorem 1 we show that such a competitive gap equilibrium must exist if none of the other non-reservation price equilibria exist. This finishes the description of the four-step procedure to construct an equilibrium.

The equilibrium construction outlined above is illustrated in Fig. 6. The Figure shows for given values of $s, \lambda$ and $\alpha$ how the equilibrium configuration depends on the cost difference $c_{H}-c_{L}$. For relatively small values of $\lambda$, Fig. 6(a) shows there are three possible equilibrium configurations, depending on whether the cost difference is small, large or intermediate. If the cost difference is relatively small, there is a unique equilibrium without a gap in the low cost distribution. When $c_{H}$ is close to $c_{L}$, the value of $\beta(\bar{p})$ has to be close to 1 and in the limit, when cost uncertainty disappears, the Stahl (1989) equilibrium is the only possible equilibrium. If, on the other hand, the cost difference $c_{H}-c_{L}$ is relatively large, then there exists a unique regular gap equilibrium. The value of $\beta(\bar{p})$ has to be relatively low to satisfy the equilibrium conditions for such an equilibrium to exist. Finally, if the cost difference $c_{H}-c_{L}$ is at intermediate values, a monopolistic gap equilibrium co-exists together with two regular gap equilibria. ${ }^{21}$ For larger values of $\lambda$, Fig. 6(b) distinguishes four possible equilibrium configurations, while equilibrium is

\footnotetext{
21 This multiplicity of equilibria is genuine and we do not know of plausible equilibrium selection arguments that can be used in this context. Fershtman and Fishman (1992) use a stability argument to argue that one of the equilibria in their search model is unstable. It is difficult to see how a stability argument can be invoked in our context, as the behavior of consumers is not characterized by a single parameter as in their model, but by the function $\beta(p)$.
} 


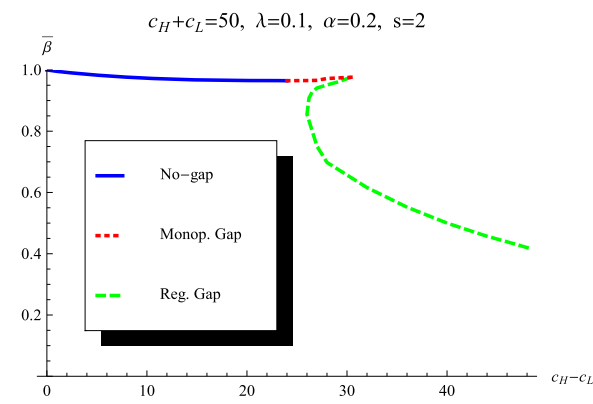

(a)

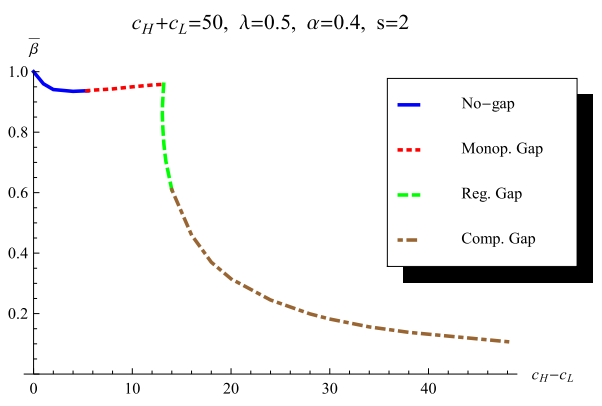

(b)

Fig. 6. $\bar{\beta}$ as a function of cost difference.

unique for each value of the cost difference. That Figure shows that a regular gap equilibrium may fail to exist if the cost difference $c_{H}-c_{L}$ is relatively large and a competitive gap equilibrium emerges.

We end this Section discussing how our analysis may shed some light on the empirical observations we mentioned at the end of the Introduction. Numerically, one can compare for a given cost realization (i) the expected first price observation conditional on the price being accepted and (ii) the expected first price observation conditional on it not being accepted. De los Santos et al. (2012) observe that in their sample the first conditional expected price is larger than the second, and they rightly claim that this is inconsistent with RPE. For the parameter values used in Figs. 3-5, one can compute and compare both conditional expected prices to conclude that for the high cost realization these non-RPE are consistent with the findings of De los Santos et al. (2012): for Fig. 4 the respective numbers are 42.94 and 42.83, for Fig. 3 the numbers are 50.81 and 49.83, while for Fig. 5 they are 45.19 and 45.11, respectively. Thus, we conclude that the observations of De los Santos et al. (2012) are not necessarily inconsistent with sequential search, although they are inconsistent with reservation price strategies.

Figs. 3, 4 and 5 show that non-RPE do not exhibit a simple monotone relationship between price and the probability of buying (or the probability of continuing to search). In a gap equilibrium, the $\beta(p)$ functions have an increasing segment, indicating that at higher prices in that segment the probability of non-shoppers buying at the firm that is visited first is higher (and thus the probability they continue searching is lower). Fig. 5 shows an extreme case of this where there is a region of prices that are set by low cost firms such that non-shoppers continue to search for sure, while at higher prices the probability of continuing to search is lower. Thus, these Figures indicate that the optimal search behavior may be highly nonmonotonic in price. De los Santos et al. (2012) empirically find that it is not the case that at higher prices, consumers are more likely to continue to search, while Honka and Chintagunta (2017) find that it is not the case that consumers with more offers in their consideration sets tend to have higher offers. Again, our analysis shows that this does not rule out that consumers search sequentially.

Finally, equilibria where the low cost price distribution has a non-convex support may be interpreted as a search theoretic foundation for the reference price principle that is discussed in marketing (see the references in the Introduction). In our model, reference prices endogenously arise from the fact that consumers rationally infer that a certain low price will only be set when cost is low, and if the common cost is really low, then the chances of finding low prices are so 


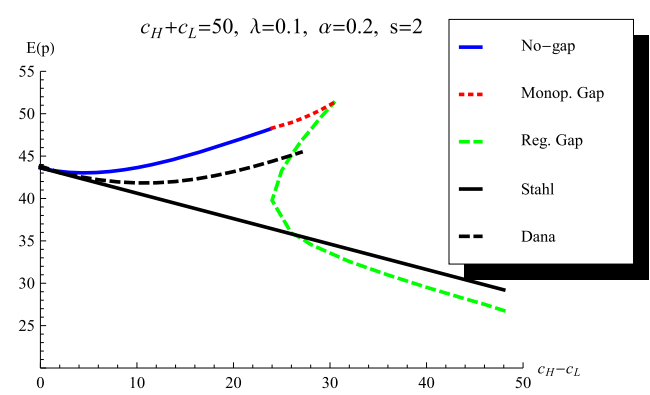

(a)

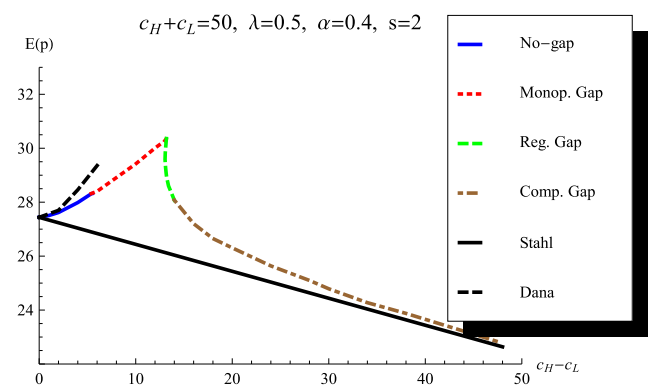

(b)

Fig. 7. Expected prices as a function of cost difference.

high that it is rational to continue searching for better deals. Thus, it is better for firms not to set prices just above these reference prices.

\section{Comparative statics and comparing models}

We are now in a position to compare the equilibrium outcomes of our model with two benchmark models, and to perform some numerical comparative statics analysis. On one hand, we use Stahl (1989) as a benchmark to show the implications of cost uncertainty. On the other hand, we use Dana (1994), or equivalently Janssen et al. (2011), as a benchmark for the outcome of RPE with cost uncertainty. As shown in Janssen et al. (2011), the expected price under RPE is larger than the weighted average of the expected price of the high and low cost equilibria as developed by Stahl (1989) and in that sense, consumers are worse off under cost uncertainty. In this Section we show that this result may well be reversed for non-RPE.

There are several effects that play a role when comparing the outcomes of non-reservation price equilibria with those of RPE. First, for a given upper bound $\bar{p}$, lowering $\beta(\bar{p})$ from an initial value of 1 (which is the value in the case of RPE) implies that there are more consumers making price comparisons. This implies firms tend to lower their prices as a reaction to the increased competition. A second effect is a direct consequence: as for a given upper bound expected prices will be lower, therefore searching for lower prices becomes more beneficial (as the expected prices after a search are lower), the upper bound has to be lower (as it is equal to the high-cost reservation price at which non-shoppers have to be indifferent between searching and buying). The third effect is that in a non-RPE, non-shoppers believe that cost is high after observing the upper bound, while in an RPE as in Dana (1994) and Janssen et al. (2011), the upper bound equals the weighted average of the reservation prices when cost is certainly low or certainly high. This effect increases the upper bound of the price distributions and thereby also the expected prices.

Fig. 7 shows the typical effect on ex ante expected price of these three effects. Expected price is a good measure of the surplus of the non-shoppers. When they continue to search, non-shoppers pay the search cost, but they also get to buy at the lowest of two prices. As in equilibrium, when they search twice they are indifferent between buying and searching, and the additional expected benefit of the possibility of buying at a lower price is exactly offset by the cost of the additional search. In both panels of Fig. 7, the average cost is taken to be 25 and the cost difference $c_{H}-c_{L}$, measured on the horizontal axis, varies between 0 (implying the cost is 
known to be 25) and 50 (where $c_{L}=0$ and $c_{H}=50$ ). When the cost difference is 0 , all models result in the same expected price. In the Stahl (1989) model where cost is known, the expected price is a fixed number larger than the cost level, where the fixed number depends on $\lambda$ and $s$, but not on $c$. The ex ante expected price reported here for the Stahl model is the weighted costs plus this fixed number. This expected price is thus decreasing in the cost difference $c_{H}-c_{L}$, if $\alpha<0.5$ (as in Fig. 7). The expected price in Dana (1994) is known to be higher than the ex ante weighted average of the expected prices in the Stahl (1989) model. The Figures also show that the RPE analyzed in Dana (1994) does not exist for larger cost differences. Figs. 7(a) and 7(b) show that for smaller cost uncertainty, expected prices are even larger than the ones reported in Janssen et al. (2011). This is due to the fact that for small cost uncertainty, the third effect outlined above dominates. For small cost uncertainty, RPE tend to underestimate firms' market power (measured by margins). The Figures also show, however, that for larger cost differences the expected price in a non-RPE becomes smaller and that it can even become smaller than the ex ante weighted average of expected prices in the Stahl (1989) model. For small values of $\lambda$, Fig. 7(a) shows that this difference can be as large as $10 \%$ ! Cost uncertainty leads here to lower market prices due to the additional search effect resulting in increased competition between firms. In Fig. 7(b), for large cost differences, the expected price converges to the ex ante expected price in the Stahl (1989) model. For large cost uncertainty, RPE may thus overestimate the market power due to search frictions.

For better understanding of the mechanism behind the comparison with the Stahl (1989) model, consider again Fig. 4 and keep in mind that Janssen et al. (2011) have shown that in the Stahl model with known cost expected price is simply a mark-up of $s /(1-\tau)$ above marginal cost, with $\tau=\int_{0}^{1} \frac{1}{1+\frac{\lambda N}{1-\lambda} \ln \frac{1+\lambda}{1-\lambda}}$ and $N=2$. For the parameters considered in Fig. 4, this mark-up approximately equals 12 so that expected prices in the two states would be 22 and 52, respectively. One can clearly see in Fig. 4 that the expected price (and margin) in the high cost state is significantly lower, while it is significantly higher in the low cost state. Low cost firms can raise prices by pretending to be high cost firms. In non-RPE this leads, however, to active consumer search from which the high cost firms suffer. The high cost margin reduces to 2.88 (from 12), while the low cost margin increases to 20.17. As $\alpha=0.5$ in Fig. 4, the average margin is smaller under cost uncertainty in a non-RPE. The potential strength of the additional search effect can be illustrated by comparing the same effects in Fig. 3 that is drawn for the same parameter values. In the monopolistic gap equilibrium in Fig. 3, prices are much higher because non-shoppers almost do not search (and when they do at prices just above $\underline{p}_{H}$ this almost does not affect the high cost distribution, as at these prices it is anyway very likely that searching consumers return to the shop to buy).

In the different panels of Fig. 8, we perform a numerical comparative static analysis showing how expected price and the probability that non-shoppers search twice, which is given by

$$
E(1-\beta(p))=\alpha \int_{\underline{p}_{H}}^{\rho_{H}}(1-\beta(p)) f_{H}(p) d p+(1-\alpha) \int_{\underline{p}_{L}}^{\rho_{H}}(1-\beta(p)) f_{L}(p) d p,
$$

changes with the changes in the different exogenous parameters $s, \lambda$ and $a$.

The first two panels (8(a) and 8(b)) show the dependence on search cost. For small search cost, a large fraction of non-shoppers performs two searches and the expected price is close to the average marginal cost of 25 . When the search cost increases from initially low levels, the expected price increases and the fraction of non-shoppers performing two searches decreases (giving firms 


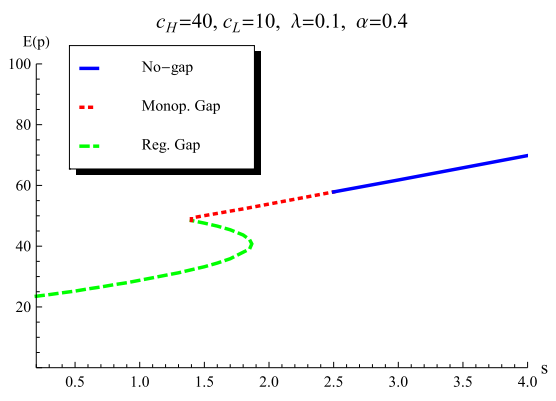

(a)

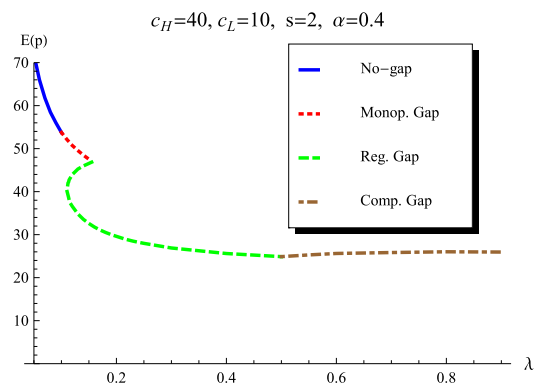

(c)

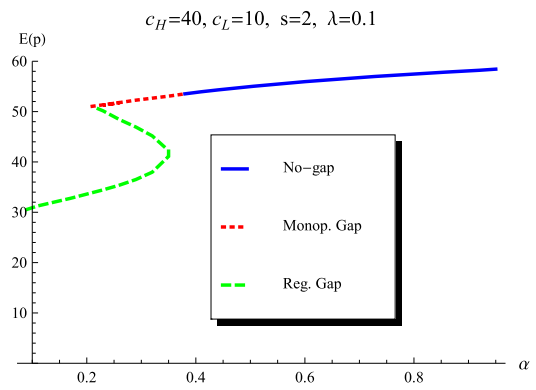

(e)

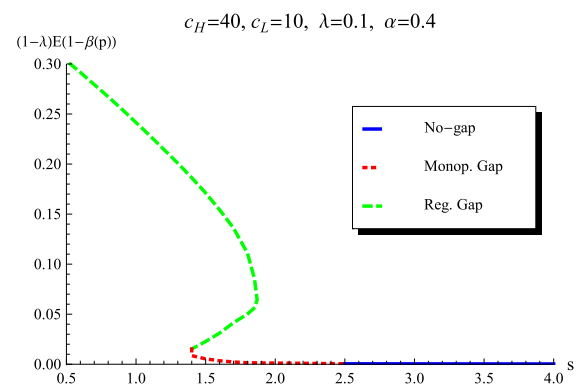

(b)

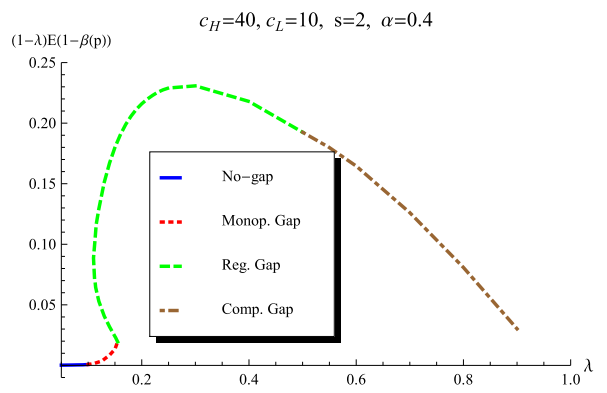

(d)

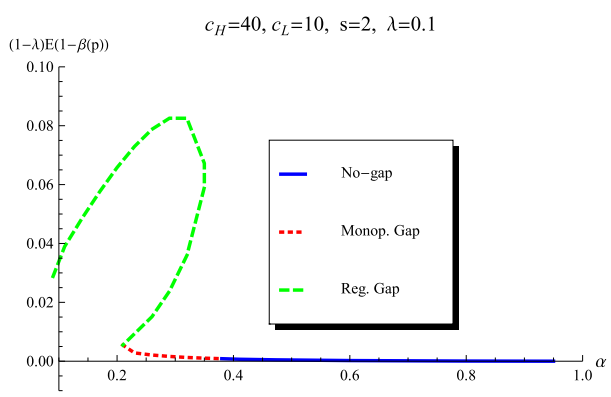

(f)

Fig. 8. Comparative statics.

more market power). At search cost levels close to 2, there are multiple gap equilibria, and it may be that the expected price is decreasing in search cost. When the search cost further increases, a no-gap equilibrium emerges and the probability of non-shoppers searching twice becomes very close to 0 . Panel (8(b)) also shows that starting from an initially small search cost, non-shoppers will search less when the search cost increases. In this way, non-shoppers partially mitigate the increase in market power typically associated with higher search cost.

The middle two panels (8(c) and 8(d)) show the dependence on the fraction of shoppers. When $\lambda$ is small, there are many non-shoppers and a no-gap equilibrium exists. In such an equilibrium, 
very few non-shoppers perform two searches and the expected price is high. When $\lambda$ increases, the expected price decreases, but in the area where multiple equilibria exist, the difference in the expected price can be quite large as the fraction of non-shoppers performing two searches differs greatly between the different equilibria. When $\lambda$ increases further, we enter the area where only competitive gap equilibria exist. In this case, increasing $\lambda$ leads to a higher probability that low cost firms price in the area where $\beta(p)=0$ and the average price increases slightly.

The last two panels (8(e) and 8(f)) show the dependence on the probability that the cost is high. When this probability is high, there is a no-gap equilibrium and consumers search very little, since there is a low probability of obtaining a substantially lower price. In this region the higher the $\alpha$, the higher the expected price. For lower values of $\alpha$, there is a monopolistic gap equilibrium with qualitatively similar properties. When $\alpha$ is sufficiently low, there are multiple gap equilibria and the incentives to search can be high, pushing the prices down. The expected price can be both increasing and decreasing in $\alpha$ depending on which of the regular gap equilibria is chosen.

\section{Extensions}

In this Section, we deal with two important extensions of our general analysis. The first relates to introducing more general forms of correlation between firms' costs, the second relates to a first analysis of oligopoly markets with sequential search.

\subsection{Introducing an idiosyncratic cost component}

In this extension we slightly modify the model described in Section 2 by introducing idiosyncratic cost shock in addition to the common cost shock we have analyzed so far. Suppose that each firm has a cost component $\kappa_{i}, i=L, H$, which is independent between the firms. Suppose that $\kappa_{H} \geq \kappa_{L}$ and the high cost state occurs with probability $\gamma$. The idiosyncratic cost shock is private information to the firm, and the common cost shock is, as before, known to both firms, but not to consumers. The total marginal cost of every firm is $c_{i j}=c_{i}+\kappa_{j}$ and we refer to the pricing strategy of such firm as $F_{i j}(p)$.

In Appendix C, we describe the analysis for the case where there is no uncertainty concerning the common cost component (the "pure idiosyncratic cost shock" case). The main take away from that analysis is that (for the same common cost component) a firm with a low idiosyncratic cost state randomizes prices over a support that is below and does not overlap with the support of the price distribution of a firm with a high idiosyncratic cost state.

Below we describe how we can use these results to combine them with the results we have derived in this paper under common cost uncertainty. We first characterize the distributions $F_{i H}(p)$ in the upper part of the support, i.e., for all $p \geq \underline{p}_{H H}$. From Appendix C, we know that the firms with low idiosyncratic cost will not price in this area. Adapting equation (1), when setting price $p$ firms with high idiosyncratic cost make a profit of

$$
\begin{aligned}
& {\left[\begin{array}{r}
\lambda\left(1-F_{i H}(p)\right)+\frac{1-\lambda}{2} \beta(p)+\frac{1-\lambda}{2}(1-\beta(p)) \gamma\left(1-F_{i H}(p)\right)+ \\
\left.\frac{1-\lambda}{2} \gamma \int_{p}^{\bar{p}}(1-\beta(\tilde{p})) f_{i H}(\tilde{p}) d \widetilde{p}\right]\left(p-c_{i H}\right)
\end{array}\right.}
\end{aligned}
$$


so that using the same technique as in the proof of Proposition 4, the distributions $F_{i H}(p)$ have to satisfy

$$
\begin{aligned}
-2 \gamma[1-(1-\lambda) \beta(p)] d F_{i H}+ & \\
& {\left[(1-\lambda) \beta^{\prime}(p)\left(1-\gamma+\gamma F_{i H}\right)+(1-\lambda) \beta(\bar{p}) \frac{\bar{p}-c_{i}}{\left(p-c_{i H}\right)^{2}}\right] d p=0 . }
\end{aligned}
$$

Solving for $F_{i H}(p)$ gives

$$
F_{i H}(p)=\frac{2 \gamma \sqrt{1-(1-\lambda) \beta(\bar{p})}-\int_{p}^{\bar{p}} \frac{(1-\gamma)(1-\lambda) \beta^{\prime}(\tilde{p})\left(\tilde{p}-c_{i H}\right)^{2}+(1-\lambda) \beta(\bar{p})\left(\bar{p}-c_{i H}\right)}{\left(\widetilde{p}-c_{i H}\right)^{2} \sqrt{1-(1-\lambda) \beta(\widetilde{p})}} d \tilde{p}}{2 \gamma \sqrt{1-(1-\lambda) \beta(p)}} .
$$

As in the pure common cost case studied in the main body of the paper, the upper bound $\bar{p}$ is determined purely by high common cost considerations. The same is true here, but now we have to take into account that a firm with a high common and idiosyncratic cost component does not know whether the other firm in the market has a high or a low idiosyncratic cost component. Applying the result on the determination of the reservation price for the idiosyncratic case derived in Appendix C, we have

$$
\bar{p}=\rho_{H}=\gamma E_{H H}(p)+(1-\gamma) E_{H L}(p)+s,
$$

where now $E_{H H}(p)$ is defined by probability distribution (12). To determine $\rho_{H}$, we now also need to have $E_{H L}(p)$.

Depending on which type of equilibrium we have, there are different cases to consider. For the no-gap equilibrium, we have that $E_{H L}(p)$ is determined by using (28) in Appendix C. This equilibrium holds if at prices $p<\underline{p}_{H H}$ (which are set by both $H L$ and $L H$ firms) consumers prefer to buy rather than continue to search. Depending on the parameters, however, it may well be that consumers prefer to continue to search at such prices (which is in line with the regular or the monopolistic gap equilibrium in the common cost framework). If that is the case, there will be a gap $\left(x, \underline{p}_{H}\right)$ in the $F_{L H}(p)$ distribution where $\beta(p)=0$ with $F_{H L}(x)=1$ and $\beta(x)=1$ such that consumers prefer to buy at prices $p \leq x .^{22}$

We conclude that it is entirely possible to extend the analysis in the main body of the paper and deal with situations where firms' cost has a common and an idiosyncratic component. Fig. 9 shows that if we add idiosyncratic cost uncertainty, the expected market price can be both lower and higher than without this uncertainty. The impact of idiosyncratic cost uncertainty on expected prices is very different than the impact of common cost uncertainty, as there is no consumer learning. The main effect is through the fact that the low and high cost distributions are not overlapping and that the reservation price is based on a weighted average of the expected prices of these two distributions.

\subsection{Oligopoly markets}

It is not too difficult to reformulate our analysis to an oligopoly model by replacing sequential search with "newspaper search" a la Salop and Stiglitz (1977) and Dana (1994). Under

\footnotetext{
22 The determination of $x$ is slightly more complicated than in the common cost case as now after observing $x$ the consumers should still update their beliefs taking into account that both $L H$ and $H L$ firms will choose $x$. $L H$ firms should be indifferent between charging prices $x$ and $\underline{p}_{H}$, while the $H L$ firms should (at least weakly) prefer charging $x$. In these cases, to determine $E_{H L}(p)$ (to be able to determine $\rho_{H}$ ), we should use (28), where $\underline{p}_{i H}$ is replaced by $x$.
} 


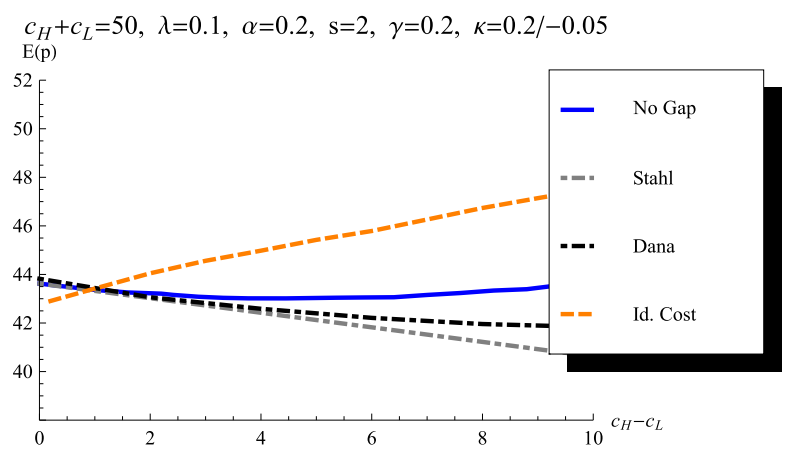

Fig. 9. Expected price in a model with idiosyncratic cost component.

newspaper search, a consumer pays a search cost only once to see all remaining prices. Thus, in both the duopoly model with sequential search and the oligopoly model with newspaper search, a consumer effectively has to take the decision whether or not to continue to search only once.

Staying in the sequential search paradigm, it is challenging to give full analytical characterization of non-RPE when there are more than two firms in the market. The difficulty is due to the fact that depending on the prices observed, consumers may perform a different number of searches, creating complications for solving for the price distribution of firms. Nevertheless, the following result on the optimal search behavior of consumers helps to considerably reduce the complexities in analyzing certain types of equilibria under oligopoly. In this result we denote by $p^{(t)}$ the price a non-shopper observes in search round $t$.

Proposition 5. Suppose the consumer was indifferent between continuing to search or buying after the first price observation $p^{(1)}$ and $f_{H}(p)>f_{L}(p)$ for all $p \in P_{(0,1)}$. Then if the consumer continued, she stops searching after the second price observation $p^{(2)}$ and buys at $\min \left\{p^{(1)}, p^{(2)}\right\}$.

There are two interesting aspects about this Proposition. First, if a non-shopper observes two prices $p^{(1)}$ and $p^{(2)}$, with $p^{(1)}<p^{(2)}$, then the Proposition says the consumer will stop searching and go back to the first firm if the high cost density is larger than the low cost density. Thus, going back to previously sampled firms before all firms are searched may well be consistent with a sequential search. De los Santos et al. (2012) have observed that consumers do go back to previously sampled firms before having visited all firms. This is inconsistent with reservation price strategies, as they noted, but not necessarily with sequential search.

Second, if in a non-RPE we have that $f_{H}(p)>f_{L}(p)$ in the price region where $\beta(p)<1$, then we know that non-shoppers will never search beyond the second firm, and the profit function under oligopoly can be written as

$$
\begin{array}{r}
\pi\left(p \mid c_{i}\right)=\left[\begin{array}{r}
\lambda\left(1-F_{i}(p)\right)^{N-1}+\frac{1-\lambda}{N} \beta(p)+\frac{1-\lambda}{N}(1-\beta(p))\left(1-F_{i}(p)\right)+ \\
\left.\frac{1-\lambda}{N-1} \frac{N-1}{N} \int_{p}^{\bar{p}}(1-\beta(\tilde{p})) f_{i}(\tilde{p}) d \tilde{p}\right]\left(p-c_{i}\right)
\end{array}\right.
\end{array}
$$




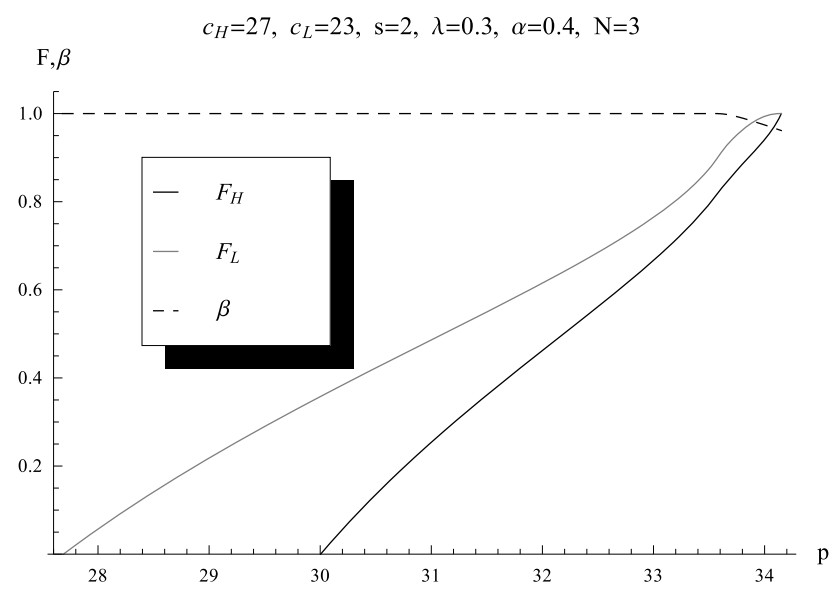

Fig. 10. Equilibrium price distributions and stopping probability for $N=3$.

so that the differential equation which has to be solved to find the distribution functions reduces to

$$
-2\left[1+\frac{\lambda N(N-1)}{2(1-\lambda)}\left(1-F_{i}\right)^{N-2}-\beta(p)\right] d F_{i}+\left[\beta^{\prime}(p) F_{i}+\beta(\bar{p}) \frac{\bar{p}-c_{i}}{\left(p-c_{i}\right)^{2}}\right] d p=0
$$

This differential equation can be solved numerically, and it can be checked whether $f_{H}(p)>$ $f_{L}(p)$ indeed holds for all prices in the price region where $\beta(p)<1$. In Fig. 10, we illustrate the distribution functions that solve (13) for particular parameter values. It can be checked that the condition on the density functions is satisfied.

In Section 4, we have seen that in the equilibria with a gap in the low cost price distribution (e.g. one depicted in Fig. 3), $f_{H}(p)<f_{L}(p)$ holds for prices close to $\underline{p}_{H}$. This implies that Proposition 5 does not hold. In that case, it may well be that with $N$ firms in the market, consumers search three or more firms before going back to the lowest price in their sample. This makes the analysis, however, quite tedious, and an analytical treatment of non-RPE is then certainly not feasible.

\section{Discussion and conclusion}

In this paper we have considered consumer search markets where firms' underlying common cost is unknown to consumers. If consumers do not know the prices different firms charge, it is natural that they also do not know the underlying cost. We have argued that in this environment of cost uncertainty, the standard RPE considered in the consumer search literature suffer from severe limitations. It was already known that RPE do not always exist, but we add that RPE implicitly assume specific out-of-equilibrium beliefs that do not satisfy standard game theoretic refinements. We characterize non-RPE that do not depend on specific assumptions regarding out-of-equilibrium beliefs and show that these equilibria always exist. Non-RPE may provide a significantly different assessment of the market power firms derive from search frictions. 
In non-RPE, non-shoppers are indifferent between buying and continuing to search over a range of prices. As prices in this range are set with positive probability, these non-RPE have active search with positive probability in equilibrium. Thus, we extend the Rothschild (1974) finding by showing in a model with endogenous price setting that in equilibrium firms price in such a way that consumers do not choose reservation price strategies. The fact that consumers rationally search more with cost uncertainty in non-RPE explains why market power may be overestimated in RPE. The additional search has a quantitatively important pro-competitive effect on prices.

Our results on non-RPE also have important consequences for the empirical literature on consumer search models that has recently taken off. Non-RPE may explain the observations of De los Santos et al. (2012) and Honka and Chintagunta (2017), as in these equilibria (i) consumers may rationally continue to search at lower prices, while they buy at higher prices and (ii) consumers may stop searching and buy at a previously visited store, before they have observed all prices in the market (see our oligopoly extension in Section 6). Moreover, the price distributions of non-RPE are quite different from the regular price distributions found in RPE. It would be interesting to see whether these price distributions provide a good fit with empirical data.

As a first inquiry into non-RPE, we have analyzed a stylized model limiting the immediate applicability of this paper to real world markets. ${ }^{23}$ In extensions, we have shown that some of the equilibria extend to oligopoly markets, and, importantly, we have dealt with markets where firms' cost consists of an idiosyncratic and a common cost component. Obviously, in an oligopoly framework one may want to consider a continuum of possible cost states. Such an extension of the present paper would be important in environments where the firms' cost is determined by an upstream firm (who can choose a continuum of different price levels). In such an environment, Janssen and Shelegia (2015a) have characterized interesting properties of RPE, but they also show such equilibria do not always exist. Non-RPE would solve this non-existence issue and it is natural to inquire into the qualitative properties of such equilibria. Bagwell and Lee (2014) provide such an analysis for the case where cost has an idiosyncratic component only. An obvious next step is to see whether our analysis on learning about a common cost component can be combined with their analysis.

One important issue that needs to be addressed in the generalizations to oligopoly markets is how consumer inferences after observing two (or more) prices interact with the consumer search decisions. In the oligopoly extension analyzed in this paper, we dealt with the easiest of different possible cases that can arise. In general, however, different possible search behaviors interact in a complicated way with the incentive of firms to choose different prices. This paper made a first step analyzing non-RPE. There are many theoretical and empirical challenges that lie ahead.

\section{Appendix A. Proofs of lemmas, propositions and corollaries}

Proposition 1. If $c_{H}-c_{L}$ is sufficiently large, or $s$ is sufficiently small an RPE does not exist.

\footnotetext{
23 Parakhonyak and Sobolev (2015) have taken a different line and introduce model uncertainty into the consumer search framework. Consumers do not update beliefs, but choose a stopping rule that minimizes their losses relative to a Bayesian consumer. They show that in such a framework, consumers also choose a non-reservation price strategy and that firms choose prices in a similar way as we have analyzed here.
} 
Proof. Our proof relies on some facts first derived in Janssen et al. (2011), which we first replicate here. The expected price $E\left(p \mid c_{i}\right)$ is given by

$$
E\left(p \mid c_{i}\right)=\int_{\underline{p}}^{\rho} p f\left(p \mid c_{i}\right) d p=c_{i}+\int_{0}^{1}\left(p-c_{i}\right) d F\left(p \mid c_{i}\right) .
$$

Introducing

$$
z \equiv 1-F\left(p \mid c_{i}\right)=\left(\frac{1-\lambda}{2 \lambda} \frac{\rho-c_{i}}{p-c_{i}}\right),
$$

we have that

$$
p-c_{i}=\frac{\rho-c_{i}}{1+\frac{2 \lambda}{1-\lambda} z} .
$$

This allows us to rewrite expression $E\left(p \mid c_{i}\right)$ as

$$
E\left(p \mid c_{i}\right)=(1-\gamma) c_{i}+\gamma \rho
$$

where $\gamma \equiv \int_{0}^{1} \frac{1}{1+\frac{2 \lambda}{1-\lambda} z} d z \in[0,1]$. As this also yields $E(p \mid \rho)=(1-\gamma) E(c \mid \rho)+\gamma \rho$, combining with $\rho=E(p \mid \rho)+s$, the reservation price is implicitly defined by

$$
\rho=E(c \mid \rho)+\frac{s}{1-\gamma}
$$

so that

$$
E\left(p \mid c_{i}\right)=c_{i}+\frac{\gamma}{1-\gamma} s+\gamma\left[E(c \mid \rho)-c_{i}\right] .
$$

Note that a high cost firm charges prices $p \geq c_{H}$, and therefore in any RPE it must be the case that $\rho \geq c_{H}$. From the characterization of RPE it is clear that all prices $p \in\left[\underline{p}{ }_{L}, \rho\right]$ are in the support of the equilibrium price distributions. Consider then a consumer who observes a price $p$ $=c_{H}-\varepsilon$ for some $\varepsilon>0$. From the expression $\underline{p}_{L}=\frac{2 \lambda}{1+\lambda} c_{i}+\frac{1-\lambda}{1+\lambda} \bar{p}$ and the fact that as shown in (14) $\bar{p}=\rho=E(c \mid \rho)+\frac{s}{1-\gamma} \leq c_{H}+\frac{s}{1-\gamma}$ it follows that $\frac{p}{L}<c_{H}$ for $c_{H}-c_{L}$ sufficiently large. Thus, observing a price $p=c_{H}-\varepsilon$ a consumer must believe that this price is set by a low cost firm. If the consumer continues to search the expected cost of buying is certainly smaller than $E\left(p \mid c_{L}\right)+s$. Then, from (15) the expected price conditional on cost being low equals

$$
E\left(p \mid c_{L}\right)=c_{L}+\frac{\gamma}{1-\gamma} s+\gamma\left[E(c \mid \bar{p})-c_{L}\right] .
$$

As $E(c \mid \bar{p})<c_{H}$, it is certainly optimal to continue searching after observing such a price (contradicting the reservation price property) if

$$
c_{L}+\frac{\gamma}{1-\gamma} s+\gamma\left(c_{H}-c_{L}\right)+s<c_{H}-\varepsilon .
$$

This reduces to

$$
\frac{1}{1-\gamma} s+\varepsilon<(1-\gamma)\left(c_{H}-c_{L}\right) .
$$

It is clear that for a given $\gamma$ and $s$, this inequality holds for $c_{H}-c_{L}$ being sufficiently large, or that for a given $\gamma$ and $c_{H}-c_{L}$ this inequality holds for some $\varepsilon>0$ for $s$ being sufficiently small. 
Proposition 2. For any search cost $s>0$, if $c_{H}-c_{L}$ is sufficiently large, then consumers search with positive probability in any PBE with continuous $F_{i}(\cdot)$.

Proof. Suppose there is no active search, i.e. $\beta(p)=1$ for all $\left\{p: p \in \operatorname{Supp} F_{L} \cup \operatorname{Supp} F_{H}\right\}$. In the proof of Proposition 1, we showed that for any $s$ if $c_{H}-c_{L}$ is large enough we have $\rho_{L}<\underline{p}$ for $\rho_{L} \leq E\left(p \mid c_{L}\right)+s .{ }^{24}$ Moreover, as $\beta(p)=1$ for all $p \leq \rho_{L}$ the price distribution $F_{L}(p)$ cannot have any mass points at these prices. As for all $p \in \operatorname{Supp} F_{L} \cup \operatorname{Supp} F_{H}$ with $p<\underline{p}_{H}$ consumers infer that cost is low, prices $p \in\left(\rho_{L}, \underline{p}_{H}\right)$ cannot be part of an equilibrium without active search. As there cannot be a mass point in the high cost price distribution at $\underline{p}_{H}$ (as otherwise high cost firms will undercut), there cannot be a masspoint in the low cost price distribution either (as consumers would infer that cost is low with probability 1$)$. This implies that $F_{L}\left(\rho_{L}\right)=F_{L}\left(p_{H}\right)$ and because $\beta\left(\rho_{L}\right)=\beta\left(\underline{p}_{H}\right)=1$ we would obtain that

$$
\left\{\lambda\left[1-F_{L}\left(\rho_{L}\right)\right]+\frac{1-\lambda}{2}\right\}\left(\rho_{L}-c_{L}\right)=\left\{\lambda\left[1-F_{L}\left(\rho_{L}\right)\right]+\frac{1-\lambda}{2}\right\}\left(\underline{p}_{H}-c_{L}\right)
$$

which is not possible since $\underline{p}_{H}>\rho_{L}$.

Proposition 3. All perfect Bayesian equilibria in which non-shoppers buy with probability one in the first search round and in which $F_{i}(p)$ is continuous, do not satisfy the D1 logic.

Proof. As only non-shoppers buy at the upper bound, in an equilibrium without active search the profits of low and high cost firms are given by $\pi_{L}=\frac{1-\lambda}{2}\left(\rho-c_{L}\right)$ and $\pi_{H}=\frac{1-\lambda}{2}\left(\rho-c_{H}\right)$, respectively. If non-shoppers buy with probability $\beta(p)$ after observing an out-of-equilibrium price $p=\rho+\epsilon$, for small $\epsilon>0$, then the deviating firm sells to $\frac{1-\lambda}{2} \beta(p)$ consumers and makes a profit of $\pi_{i}=\frac{1-\lambda}{2} \beta(p)\left(p-c_{i}\right), i=L, H$ as shoppers will not buy at these high deviation prices. This deviation profit is larger than the equilibrium profit if

$$
\beta(p)>\frac{\rho-c_{i}}{p-c_{i}} .
$$

Thus, for an out-of-equilibrium price $p=\rho+\epsilon$, for small enough $\epsilon>0$, the set $\left\{q_{i} \in B_{i}(p)\right.$ : $\left.\left(p-c_{i}\right) q_{i} \geq \pi_{i}^{i *}\right\}=\left(\frac{1-\lambda}{2} \frac{\rho-c_{i}}{p-c_{i}}, \frac{1-\lambda}{2}\right]$. As the lower bound of this set is decreasing in $c_{i}$ for all $p>\rho,\left\{q_{i} \in B_{i}(p):\left(p-c_{L}\right) q_{i} \geq \pi_{L}^{i *}\right.$ and $\left.p>\rho\right\} \subset\left\{q_{i} \in B_{i}(p):\left(p-c_{i}\right) q_{H} \geq \pi_{H}^{i *}\right.$ and $\left.p>\rho\right\}$. The D1 refinement thus requires that $\operatorname{Pr}\left(c_{L} \mid p\right)=0$ for all $p>\rho$.

The remaining part of the proof is given in the main text just above the Proposition.

Lemma 1. In PBE with continuous $F_{i}(p)$, thus, in any non-RPE, $\bar{p}_{L}=\bar{p}_{H} \equiv \bar{p}$.

Proof. If the upper bounds are not equal, it must be the case that $\bar{p}_{H}>\bar{p}_{L}$, or vice versa. As the argument in both cases is identical, we only consider the case where $\bar{p}_{H}>\bar{p}_{L}$. Due to the fact that the price distributions do not have mass points, it must be the case that in a left neighborhood of $\bar{p}_{H}$ high cost firms charge prices with strictly positive probability. For any small

\footnotetext{
24 Although formally this result was shown only for RPE, it is clear that in any equilibrium without active search (i.e., where consumers buy immediately at the first search) it must hold. Indeed, it follows form the fact that $\underline{p}_{L}=$ $\frac{2 \lambda}{1+\lambda} c_{i}+\frac{1-\lambda}{1+\lambda} \bar{p}$, which in turn follows from the equal-profit condition in the case when all consumers buy at $\underline{p} L$ and $\bar{p}$ with probability one (which is true in all equilibria without active search) and the fact that $\underline{p}_{H} \geq c_{H}$.
} 
$\varepsilon>0$ consider then the interval $\left(\bar{p}_{H}-\varepsilon, \bar{p}_{H}\right)$. If a low cost firm would not charge prices in this interval, consumers would know that cost is high after observing prices in this interval. Given that consumers are (at least) indifferent between buying and not buying at $\bar{p}_{H}$ (as, if consumers prefer to continue to search after observing $\bar{p}_{H}$, no firm would ever charge $\bar{p}_{H}$ ), they strictly prefer to buy at prices in the interval $\left(\bar{p}_{H}-\varepsilon, \bar{p}_{H}\right)$. But then low cost firms would prefer to set prices in this interval as well instead of charging $\bar{p}_{L}$. Thus, $\bar{p}_{L}=\bar{p}_{H}$.

Proposition 4. If $F(p)$ is a price distribution in a non-RPE, then it should be of the following form:

$$
F_{i}(p)= \begin{cases}\frac{2 \sqrt{1-(1-\lambda) \beta(\bar{p})}-\int_{p}^{\bar{p}} \frac{(1-\lambda) \beta(\bar{p})\left(\bar{p}-c_{i}\right)}{\left(\widetilde{p}-c_{i}\right)^{2} \sqrt{1-(1-\lambda) \beta(\widetilde{p})}} d \tilde{p}}{2 \sqrt{1-(1-\lambda) \beta(p)}} & \text { if } p \in P_{(0,1)} \\ 1-\frac{1-\lambda}{2 \lambda}\left[\beta(\bar{p}) \frac{\bar{p}-c_{i}}{p-c_{i}}-1-\int_{p}^{\bar{p}}(1-\beta(\widetilde{p})) f_{i}(\widetilde{p}) d \widetilde{p}\right] & \text { if } p \in P_{1} \\ 1-\frac{1-\lambda}{1+\lambda}\left[\beta(\bar{p}) \frac{\bar{p}-c_{i}}{p-c_{i}}-\int_{p}^{\bar{p}}(1-\beta(\tilde{p})) f_{i}(\tilde{p}) d \tilde{p}\right] & \text { if } p \in P_{0}\end{cases}
$$

Proof. Assuming the function $\beta(p)$ is differentiable, equation (6) can be rewritten as

$$
-2[1-(1-\lambda) \beta(p)] f_{i}(p)+(1-\lambda) \beta^{\prime}(p) F_{i}(p)=-(1-\lambda) \beta(\bar{p}) \frac{\bar{p}-c_{i}}{\left(p-c_{i}\right)^{2}}
$$

by taking the derivative of both sides of the equality sign. This equation can be explicitly written as a differential equation:

$$
-2[1-(1-\lambda) \beta(p)] d F_{i}+\left[(1-\lambda) \beta^{\prime}(p) F_{i}+(1-\lambda) \beta(\bar{p}) \frac{\bar{p}-c_{i}}{\left(p-c_{i}\right)^{2}}\right] d p=0 .
$$

As

$$
-2 \frac{\partial[1-(1-\lambda) \beta(p)]}{\partial p} \neq \frac{\partial\left[(1-\lambda) \beta^{\prime}(p) F_{i}+(1-\lambda) \beta(\bar{p}) \frac{\bar{p}-c_{i}}{\left(p-c_{i}\right)^{2}}\right]}{\partial F_{i}}
$$

this is an inexact linear differential equation. However, it can be made exact by dividing (16) by $\sqrt{1-(1-\lambda) \beta(p)}$ :

$$
-2 \sqrt{1-(1-\lambda) \beta(p)} d F_{i}+\frac{\left[(1-\lambda) \beta^{\prime}(p) F_{i}+(1-\lambda) \beta(\bar{p}) \frac{\bar{p}-c_{i}}{\left(p-c_{i}\right)^{2}}\right]}{\sqrt{1-(1-\lambda) \beta(p)}} d p=0 .
$$

The solution to this exact differential function is a function $Z\left(F_{i}, p\right)=C_{i}$ (where $C_{i}$ is an integration constant) with $\frac{\partial Z}{\partial p}=\frac{\left[(1-\lambda) \beta^{\prime}(p) F_{i}+(1-\lambda) \beta(\bar{p}) \frac{\bar{p}-c_{i}}{\left(p-c_{i}\right)^{2}}\right]}{\sqrt{1-(1-\lambda) \beta(p)}}$ and $\frac{\partial Z}{\partial F_{i}}=\sqrt{1-(1-\lambda) \beta(p)}$. It follows that the solution $Z\left(F_{i}, p\right)$ is given by

$$
-2 F_{i} \sqrt{1-(1-\lambda) \beta(p)}+\int \frac{(1-\lambda) \beta(\bar{p})\left(\bar{p}-c_{i}\right)}{\left(p-c_{i}\right)^{2} \sqrt{1-(1-\lambda) \beta(p)}} d p+C_{i}=0 .
$$

This equation can be solved explicitly for $F_{i}(p)$, to yield (7), where the integration constant $C_{i}$ is found by setting $F_{i}(\bar{p})=1$.

If $\beta(p)=1$ or $\beta(p)=0$ in an interval of prices $(\widehat{p}, \widetilde{p})$, then the equilibrium price distribution can be simply directly calculated from (6). 
Corollary 3. In any non-RPE, for all $p<\bar{p}, F_{L}(p) \geq F_{H}(p)$ and whenever $0<F_{H}(p)<1$, $F_{L}(p)>F_{H}(p)$.

Proof. From the previous Proposition, it follows that $F_{H}(p)<F_{L}(p)$ if, and only if, $\frac{(1-\lambda) \beta(\bar{p})\left(\bar{p}-c_{H}\right)}{\left(p-c_{H}\right)^{2} \sqrt{1-(1-\lambda) \beta(p)}}>\frac{(1-\lambda) \beta(\bar{p})\left(\bar{p}-c_{L}\right)}{\left(p-c_{L}\right)^{2} \sqrt{1-(1-\lambda) \beta(p)}}$ for all $p$. This is the case if

$$
\left(p-c_{H}\right)^{2}\left(\bar{p}-c_{L}\right)<\left(p-c_{L}\right)^{2}\left(\bar{p}-c_{H}\right) .
$$

This can be rewritten as

$$
\left(c_{H}-c_{L}\right) p^{2}-\left(\left(c_{H}-c_{L}\right) \bar{p} p+c_{L} c_{H}\left(c_{L}-c_{H}\right)<0\right.
$$

or $p^{2}-\bar{p} p-c_{L} c_{H}<0$, which is definitely the case as $p<\bar{p}$.

Lemma 2. Let $p^{*}$ be such that $\beta\left(p^{*}\right)=1$ and for any sufficiently small $\epsilon>0 \beta\left(p^{*}+\epsilon\right)<1$. Suppose that $p^{*}$ is in the interior of the support of $F_{i}(p), i=L, H$. Then in equilibrium it must be that $\beta^{\prime}\left(p^{*}\right)=0$.

Proof. Suppose, $\beta^{\prime}\left(p^{*}\right)<0$. Denote $\Delta f_{i}=f_{i}\left(p^{*}-\varepsilon\right)-f_{i}\left(p^{*}+\varepsilon\right)$. Then, since

$$
f_{i}(p)=\frac{(1-\lambda) \beta^{\prime}(p) F_{i}(p)+(1-\lambda) \beta(\bar{p}) \frac{\bar{p}-c_{i}}{\left(p-c_{i}\right)^{2}}}{2[1-(1-\lambda) \beta(p)]}
$$

we have

$$
\begin{aligned}
& \Delta f_{i}=\frac{1}{2}\left(\frac{\beta(\bar{p})\left(\bar{p}-c_{i}\right)(1-\lambda)}{\left(p^{*}-\varepsilon-c_{i}\right)^{2} \lambda}-\frac{(1-\lambda)\left(\frac{\beta(\bar{p})\left(\bar{p}-c_{i}\right)}{\left(p^{*}+\varepsilon-c_{i}\right)^{2}}+F_{i}\left(p^{*}+\varepsilon\right) \beta^{\prime}\left(p^{*}+\varepsilon\right)\right)}{1-(1-\lambda) \beta\left(p^{*}+\varepsilon\right)}\right)= \\
& =\frac{1}{2}\left(\frac{\beta(\bar{p})\left(\bar{p}-c_{i}\right)(1-\lambda)}{\left(p^{*}-\varepsilon-c_{i}\right)^{2} \lambda}-\frac{(1-\lambda)\left(\frac{\beta(\bar{p})\left(\bar{p}-c_{i}\right)}{\left(p^{*}+\varepsilon-c_{i}\right)^{2}}\right)}{1-(1-\lambda) \beta\left(p^{*}+\varepsilon\right)}\right)-\frac{F_{i}\left(p^{*}+\varepsilon\right) \beta^{\prime}\left(p^{*}+\varepsilon\right)}{1-(1-\lambda) \beta\left(p^{*}+\varepsilon\right)}
\end{aligned}
$$

As $F_{L}(p)>F_{H}(p)$ and $\left\{\frac{\bar{p}-c_{i}}{\left(p-c_{i}\right)^{2}}\right\}_{c_{i}}^{\prime}>0$, we get that $f_{L}, f_{H}>0$ for any $\varepsilon$. Now we take a limit with respect to $\varepsilon$.

$$
\lim _{\varepsilon \rightarrow 0} \Delta f_{i}=0+\lim _{\varepsilon \rightarrow 0}-\frac{F_{i}\left(p^{*}+\varepsilon\right) \beta^{\prime}\left(p^{*}+\varepsilon\right)}{1-(1-\lambda) \beta\left(p^{*}+\varepsilon\right)}
$$

The first term in equation (17) approaches zero when $\varepsilon$ approaches zero, while the second is strictly positive and bounded below. So we can conclude that as $F_{L}(p)>F_{H}(p)$, we can always find sufficiently small $\varepsilon$, such that $\Delta f_{L}>\Delta f_{H}$.

Denote

$$
a_{i}=(1-\lambda) \beta(\bar{p})\left(\bar{p}-c_{i}\right)
$$

Then

$$
\frac{a_{L}}{\left(p-c_{L}\right)^{2}}<\frac{a_{H}}{\left(p-c_{H}\right)^{2}}
$$


which implies that $f_{L}<f_{H}$ for prices higher than $p^{*}$. This gives $\frac{f_{L}\left(p^{*}-\varepsilon\right)}{(1-\alpha) f_{L}\left(p^{*}-\varepsilon\right)+\alpha f_{H}\left(p^{*}-\varepsilon\right)}=$ $\frac{f_{L}\left(p^{*}+\varepsilon\right)+\Delta f_{L}}{(1-\alpha) f_{L}\left(p^{*}+\varepsilon\right)+\alpha f_{H}\left(p^{*}+\varepsilon\right)+(1-\alpha) \Delta f_{L}+\alpha \Delta f_{H}}>\frac{f_{L}\left(p^{*}+\varepsilon\right)}{(1-\alpha) f_{L}\left(p^{*}+\varepsilon\right)+\alpha f_{H}\left(p^{*}+\varepsilon\right)}$. Thus, if consumers are indifferent at $p^{*}+\varepsilon$, they must strictly prefer to continue searching at $p^{*}-\varepsilon$, which can not be the case. Therefore, $\beta^{\prime}\left(p^{*}\right)=0$ (since it cannot be greater than 0 ).

Proposition 5. Suppose the consumer was indifferent between continuing to search or buying after the first price observation $p^{(1)}$ and $f_{H}(p)>f_{L}(p)$ for all $p \in P_{(0,1)}$. Then if the consumer continued, she stops searching after the second price observation $p^{(2)}$ and buys at $\min \left\{p^{(1)}, p^{(2)}\right\}$.

Proof. Consider a consumer who has observed two prices $p^{(1)}$ and $p^{(2)}$. Given that the consumer was indifferent after observing $p^{(1)}$, the optimal stopping rule for the first round gives

$$
w_{1}\left(p^{(1)}\right)\left[\Phi_{L}\left(p^{(1)}\right)-s\right]+\left[1-w_{1}\left(p^{(1)}\right)\right]\left[\Phi_{H}\left(p^{(1)}\right)-s\right]=0,
$$

where

$$
w_{1}\left(p^{(1)}\right)=\frac{(1-\alpha) f_{L}\left(p^{(1)}\right)}{(1-\alpha) f_{L}\left(p^{(1)}\right)+\alpha f_{H}\left(p^{(1)}\right)} .
$$

After observing $p^{(2)}$ the decision of the consumer is determined by the sign of

$$
w_{2}\left(p^{(1)}, p^{(2)}\right)\left[\Phi_{L}\left(p^{(1)}\right)-s\right]+\left[1-w_{1}\left(p^{(1)}, p^{(2)}\right)\right]\left[\Phi_{H}\left(p^{(1)}\right)-s\right],
$$

where

$$
w_{2}\left(p^{(1)}, p^{(2)}\right)=\frac{(1-\alpha) f_{L}\left(p^{(1)}\right) f_{L}\left(p^{(2)}\right)}{(1-\alpha) f_{L}\left(p^{(1)}\right) f_{L}\left(p^{(2)}\right)+\alpha f_{H}\left(p^{(1)}\right) f_{H}\left(p^{(2)}\right)} .
$$

Note, that if $w_{2}\left(p^{(1)}, p^{(2)}\right)<w_{1}\left(p^{(1)}\right)$ this sign is always negative and the consumer prefers to stop. This is the case if

$$
\frac{(1-\alpha) f_{L}\left(p^{(1)}\right)}{(1-\alpha) f_{L}\left(p^{(1)}\right)+\alpha f_{H}\left(p^{(1)}\right)}>\frac{(1-\alpha) f_{L}\left(p^{(1)}\right) f_{L}\left(p^{(2)}\right)}{(1-\alpha) f_{L}\left(p^{(1)}\right) f_{L}\left(p^{(2)}\right)+\alpha f_{H}\left(p^{(1)}\right) f_{H}\left(p^{(2)}\right)},
$$

which can be rewritten as

$$
\begin{array}{r}
(1-\alpha)^{2} f_{L}\left(p^{(1)}\right) f_{L}\left(p^{(2)}\right)+(1-\alpha) \alpha f_{L}\left(p^{(1)}\right) f_{H}\left(p^{(1)}\right) f_{H}\left(p^{(2)}\right)> \\
(1-\alpha)^{2} f_{L}\left(p^{(1)}\right) f_{L}\left(p^{(2)}\right)+(1-\alpha) \alpha f_{L}\left(p^{(1)}\right) f_{H}\left(p^{(1)}\right) f_{L}\left(p^{(2)}\right),
\end{array}
$$

and reduces to

$$
f_{H}\left(p^{(2)}\right)>f_{L}\left(p^{(2)}\right) .
$$

Proposition A.1. In any non-reservation price equilibrium,

$$
\beta^{\prime}(\bar{p}) \equiv \lim _{p \uparrow \bar{p}} \frac{\beta(\bar{p})-\beta(p)}{\bar{p}-p}=-\frac{\beta(\bar{p})}{\bar{p}-c_{L}}
$$

and $\beta(\bar{p})<1$. 
Proof. As it follows from equations (8) and (9), non-shoppers have to be indifferent between buying and continuing to search after observing $\bar{p}$.

From Lemma 1 , it follows that in the interval $(\bar{p}-\varepsilon, \bar{p})$ both types of firms charge prices with strictly positive probability. By differentiating (5) with respect to $p$ and setting $f_{L}(\bar{p})=0$ and $F_{L}(\bar{p})=1$ we obtain

$$
\beta^{\prime}(\bar{p})\left(\bar{p}-c_{L}\right)+\beta(\bar{p})=0,
$$

which can only be the case when $\beta(\bar{p})<1$.

\section{Appendix B. Proof of Theorem 1}

Here, we prove the existence of equilibrium (Theorem 1) in several lemmas and a final main result. In general, and as explained in the main text, we need to prove that the two functional equations characterizing the distribution functions and the optimality condition for the search rule of non-shoppers, i.e., equations (7) and (10), have an economically meaningful solution. Thus, the distribution functions should be well-defined, i.e. the densities are positive, and $0 \leq \beta(p) \leq 1$. If $\beta(p)=0$ it should be optimal for non-shoppers to continue searching after observing these prices, while at prices where $\beta(p)=1$ non-shoppers should prefer to buy. In addition, two boundary conditions need to be satisfied and we have two parameters to satisfy them: $\bar{p}$ and $\beta(\bar{p})$. First, we need that $f_{L}(\bar{p})=0$, which implies that $\int_{0}^{\bar{p}} F_{H}(x) d x=s$. The second boundary condition is different for different parameter values. For the purpose of formulating this second boundary condition, implicitly define $p_{0} \leq \underline{p}_{H}$ as $\pi_{L}\left(p_{0}\right)=\pi_{L}\left(\underline{p}_{H}\right)$, or

$$
\begin{aligned}
& {\left[\lambda\left(1-F_{L}\left(p_{0}\right)\right)+\frac{1-\lambda}{2}+\frac{1-\lambda}{2} \int_{p_{0}}^{\underline{p}_{H}} f_{i}(\tilde{p}) d \tilde{p}+\frac{1-\lambda}{2} \int_{\underline{p}_{H}}^{\bar{p}}(1-\beta(\tilde{p})) f_{i}(\tilde{p}) d \tilde{p}\right] \times} \\
& \left(p_{0}-c_{L}\right)= \\
& {\left[\lambda\left(1-F_{L}\left(\underline{p}_{H}\right)\right)+\frac{1-\lambda}{2}-\frac{1-\lambda}{2}\left(1-\beta\left(\underline{p}_{H}\right)\right) F_{L}\left(\underline{p}_{H}\right)\right.} \\
& \left.+\frac{1-\lambda}{2} \int_{\underline{p}_{H}}^{\bar{p}}(1-\beta(\tilde{p})) f_{L}(\tilde{p}) d \tilde{p}\right]\left(\underline{p}_{H}-c_{L}\right) .
\end{aligned}
$$

That is, $p_{0}$ is the largest price smaller than $\underline{p}_{H}$ that makes low cost firms indifferent between $(i)$ setting this price and having uninformed consumers immediately buy at this price and not buying for sure at any price in the interval $\left(p_{0}, \underline{p}_{H}\right)$ and (ii) choosing $\underline{p}_{H}$ and having uninformed consumers buying with probability $\beta\left(\underline{p}_{H}\right)$. To see that $p_{0}$ is uniquely defined, consider the following two cases. If low cost firms do not charge prices in the interval $\left(p_{0}, \underline{p}_{H}\right)$ with positive probability, then the demand at $p_{0}$ is independent of $p_{0}$ and thus the profit expression is increasing in $p_{0}$. In that case, if $\beta\left(\underline{p}_{H}\right)=1$, then $p_{0}=\underline{p}_{H}$, while if $\beta\left(\underline{p}_{H}\right)<1$, then $p_{0}<\underline{p}_{H}$. If, on the other hand, with positive probability low cost firms do charge prices in the interval $\left(p_{0}, \underline{p}_{H}\right)$, then the profit at $p_{0}$ can be written as 


$$
\begin{aligned}
\pi_{L}\left(p_{0}\right)= & {\left[\frac{1+\lambda}{2}\left(1-F_{L}\left(p_{0}\right)\right)+\frac{1-\lambda}{2} F_{L}\left(\underline{p}_{H}\right)+\frac{1-\lambda}{2} \int_{\underline{p}_{H}}^{\bar{p}}(1-\beta(\tilde{p})) f_{i}(\tilde{p}) d \tilde{p}\right] } \\
& \times\left(p_{0}-c_{L}\right),
\end{aligned}
$$

which using (3) for $\beta\left(p_{0}\right)=1$, can be written as

$$
\begin{array}{r}
\frac{1+\lambda}{2}\left(\frac{1-\lambda}{2 \lambda}\left[\beta(\bar{p}) \frac{\bar{p}-c_{L}}{p_{0}-c_{i}}-1-\int_{p_{0}}^{\bar{p}}(1-\beta(\tilde{p})) f_{i}(\tilde{p}) d \widetilde{p}\right]\right)\left(p_{0}-c_{L}\right) \\
+\left(\frac{1-\lambda}{2} F_{L}\left(\underline{p}_{H}\right)+\frac{1-\lambda}{2} \int_{\underline{p}_{H}}^{\bar{p}}(1-\beta(\widetilde{p})) f_{i}(\widetilde{p}) d \tilde{p}\right)\left(p_{0}-c_{L}\right),
\end{array}
$$

or

$$
\begin{aligned}
& \frac{1-\lambda^{2}}{4 \lambda}\left[\beta(\bar{p})\left(\bar{p}-c_{L}\right)-\left(1+\int_{p_{0}}^{\bar{p}}(1-\beta(\tilde{p})) f_{i}(\tilde{p}) d \tilde{p}\right)\left(p_{0}-c_{L}\right)\right] \\
& +\left(\frac{1-\lambda}{2} F_{L}\left(\underline{p}_{H}\right)+\frac{1-\lambda}{2} \int_{\underline{p}_{H}}^{\bar{p}}(1-\beta(\tilde{p})) f_{i}(\tilde{p}) d \tilde{p}\right)\left(p_{0}-c_{L}\right),
\end{aligned}
$$

which is clearly increasing in $p_{0}$.

The second boundary condition can then be stated as follows.

(i) If $\rho_{L} \geq \underline{p}_{H}$, then $\beta^{\prime}(p)=0$ when $p$ is such that $\beta(p)=1$ (Lemma 2);

(ii) If $\beta(p)<1$ for all $p \in\left[\underline{p}_{H}, \bar{p}\right]$, then $p_{0}=\rho_{L}$;

(iii) If $\rho_{L}<\underline{p}_{H}$ and there is an interval $[x, y]$ of prices $p$ such that $\beta(p)=1$ for all $p \in[x, y]$, then $\lim _{p \downarrow y} \beta^{\prime}(p)=0$ (Lemma 2), and $p_{0}=\rho$.

To simplify notation, we rewrite the distribution functions as

$$
F_{i}(p)=\frac{2 g(\bar{p})-\int_{p}^{\bar{p}} \frac{a_{i}}{\left(x-c_{i}\right)^{2} g(x)} d x}{2 g(p)} \quad i=L, H,
$$

where $g(p)=\sqrt{1-(1-\lambda) \beta(p)}$ and $a_{i}=(1-\lambda) \beta(\bar{p})\left(\bar{p}-c_{i}\right)$, and proceed as follows. We first note that (10) and (7) only need to hold in an interval of prices where $\beta(p)<1$ and that this is a subset of $\left(\rho_{L}, \bar{p}\right.$ ]. Lemma A.1 shows that this implies that $f_{L}(p)$ and $f_{H}(p)$ are either both positive or both negative over the relevant interval. We next show that $f_{H}(\bar{p})>0$. Together with Lemma A.1, this shows that if the indifference equation for consumers has a solution, then the distribution functions in (7) are well-defined, increasing functions.

Lemma A.1. For any $p \in P_{(0,1)}, f_{L}(p) \cdot f_{H}(p) \geq 0$.

Proof. As $\Phi_{L}\left(\rho_{L}\right)=\int_{0}^{\rho_{L}} F_{i}(x) d x=\Phi_{H}\left(\rho_{H}\right)=\int_{0}^{\rho_{H}} F_{i}(x) d x=s$, and $\Phi_{i}(p)$ are increasing functions it follows that $\Phi_{L}(p)>s$ and $\Phi_{H}(p)<s$ for all $\rho_{L}<p<\rho_{H}$. As (10) can be rewritten as 


$$
\frac{(1-\alpha) f_{L}(p)}{(1-\alpha) f_{L}(p)+\alpha f_{H}(p)}\left(\Phi_{L}(p)-s\right)+\frac{\alpha f_{H}(p)}{(1-\alpha) f_{L}(p)+\alpha f_{H}(p)}\left(\Phi_{H}(p)-s\right)=0
$$

it follows that both the weights $\frac{(1-\alpha) f_{L}(p)}{(1-\alpha) f_{L}(p)+\alpha f_{H}(p)}$ and $\frac{\alpha f_{H}(p)}{(1-\alpha) f_{L}(p)+\alpha f_{H}(p)}$ have to be of the same sign, which can only be the case if $f_{L}(p)$ and $f_{H}(p)$ have the same sign.

Lemma A.2. For all $p \in\left[\max \left(\rho_{L}, \underline{p}_{H}\right), \bar{p}\right] \cap P_{(0,1)}, f_{i}(p)>0, i=L, H$.

Proof. As the function $\beta(p)$ is differentiable, equation (6) can be rewritten as

$$
-2[1-(1-\lambda) \beta(p)] f_{i}(p)+(1-\lambda) \beta^{\prime}(p) F_{i}(p)=-(1-\lambda) \beta(\bar{p}) \frac{\bar{p}-c_{i}}{\left(p-c_{i}\right)^{2}},
$$

which at $\bar{p}$ reduces to

$$
-2[1-(1-\lambda) \beta(\bar{p})] f_{i}(\bar{p})=-(1-\lambda)\left[\frac{\beta(\bar{p})}{\bar{p}-c_{i}}+\beta^{\prime}(\bar{p})\right] .
$$

As the RHS of this expression equals 0 for $c_{i}=c_{L}$, the RHS is clearly negative for $c_{i}=c_{H}$ for any choice of $0<\beta(\bar{p})<1$. Thus, $f_{H}(\bar{p})>0$. By continuity, there exists $\varepsilon>0$ such that for all $p \in[\bar{p}-\varepsilon, \bar{p}] f_{H}(p)>0$. Then, by Lemma A.1 $f_{L}(\cdot)$ is also positive in the interior of this interval. Moreover, Lemma A.1 implies that if $f_{L}(\cdot)$ and $f_{H}(\cdot)$ change sign it must happen at the same price, which we denote as $q \in\left[\max \left(\rho_{L}, \underline{p}_{H}\right), \bar{p}\right]$. By differentiating (7) and taking the ratio of the derivatives, we obtain

$$
\frac{\left(\bar{p}-c_{H}\right)\left(q-c_{L}\right)^{2}}{\left(q-c_{H}\right)^{2}\left(\underline{p}-c_{L}\right)}=\frac{F_{H}(q)}{F_{L}(q)} .
$$

Note, that the LHS of this expression is larger than 1 (since $q<\bar{p}$ ), while by Corollary 3 the RHS is smaller than 1 . Therefore, there is no such $q$ and both densities must be positive.

Thus, it directly follows from Lemma A.1 and A.2 that both density functions have to be positive for all $p \in\left[\max \left(\rho_{L}, \underline{p}_{H}\right), \bar{p}\right] \cap P_{(0,1)}$. As for all other prices $\beta(p)=0$ or $\beta(p)=1$, the density functions are positive for all prices.

We also need that consumers prefer to buy as long as $\beta(p)=1$. The proof is a simple adaptation of a proof given by Dana (1994) that in a reservation price equilibrium uninformed consumers strictly prefer to buy at all prices in the support of the price distribution of the high cost firm that are strictly smaller than the reservation price.

Lemma A.3. If $\beta(p)=1$ on a certain interval $[x, y]$ and uninformed consumers weakly prefer buying to continuing searching at $p=y$, then these consumers strictly prefer buying to continuing searching at $p \in[x, y)$.

Proof. If $\beta(p)=1$, then

$$
\frac{f_{H}(p)}{f_{L}(p)}=\frac{\left(p-c_{H}\right)^{2}\left(\bar{p}-c_{L}\right)}{\left(p-c_{L}\right)^{2}\left(\bar{p}-c_{H}\right)} .
$$

This expression is decreasing in $p$. Thus, after observing a larger price, updating beliefs results in uninformed consumers believing it is more likely that cost is high. The expected pay-off of continuing to search is thus larger at larger prices. At the same time, the pay-off of buying at a 
higher price decreases. Thus, if a consumer is indifferent between the two options at $p=x$, then she must strictly prefer buying at $p<x$.

We also need that along the equilibrium path we construct, consumers prefer to continue searching when $\beta(p)=0$. This is, however, trivial, as $\beta(p)=0$ only occurs along the equilibrium path when $\rho_{L}<p<\underline{p}_{H}$, but in that case consumers infer that it is only low cost firms that charge such prices, and non-shoppers prefer to search on as these prices are above $\rho_{L}$.

The next five lemmas establish that our system can be rewritten into five proper differential equations and invoke the Pickard-Lindelof theorem of differential equations to show that the system has a (mathematical) solution that it is locally unique. To make sure that the conditions of this theorem apply, we first need to establish some properties of the function $g^{\prime}(p)$.

Lemma A.4. The solution to the indifference equation (10) can be written as

$$
g^{\prime}(p)=\frac{(1-\alpha) \frac{a_{L}}{2\left(p-c_{L}\right)^{2} g(p)}\left(\Phi_{L}(p)-s\right)+\alpha \frac{a_{H}}{2\left(p-c_{H}\right)^{2} g(p)}\left(\Phi_{H}(p)-s\right)}{(1-\alpha) F_{L}(p)\left(\Phi_{L}(p)-s\right)+\alpha F_{H}(p)\left(\Phi_{H}(p)-s\right)} .
$$

Proof. Taking the derivative of (19) gives

$$
f_{i}(p)=\frac{1}{g(p)}\left(\frac{a_{i}}{2\left(p-c_{i}\right)^{2} g(p)}-F_{i}(p) g^{\prime}(p)\right) .
$$

Then the optimal stopping rule can be rewritten as

$$
\begin{aligned}
0= & \frac{(1-\alpha)\left(\frac{a_{L}}{2\left(p-c_{L}\right)^{2} g(p)}-F_{L}(p) g^{\prime}(p)\right)\left(\Phi_{L}(p)-s\right)}{(1-\alpha)\left(\frac{a_{L}}{2\left(p-c_{L}\right)^{2} g(p)}-F_{L}(p) g^{\prime}(p)\right)+\alpha\left(\frac{a_{H}}{2\left(p-c_{H}\right)^{2} g(p)}-F_{H}(p) g^{\prime}(p)\right)} \\
& +\frac{\alpha\left(\frac{a_{H}}{2\left(p-c_{H}\right)^{2} g(p)}-F_{H}(p) g^{\prime}(p)\right)\left(\Phi_{H}(p)-s\right)}{(1-\alpha)\left(\frac{a_{L}}{2\left(p-c_{L}\right)^{2} g(p)}-F_{L}(p) g^{\prime}(p)\right)+\alpha\left(\frac{a_{H}}{2\left(p-c_{H}\right)^{2} g(p)}-F_{H}(p) g^{\prime}(p)\right)}
\end{aligned}
$$

which can easily be rewritten as the equation in the statement of the Lemma.

We proceed with some facts about the function $g^{\prime}(p)$. Define $g^{\prime}(p)=\frac{A(p)}{B(p)}$, where

$$
A(p) \equiv(1-\alpha) \frac{a_{L}}{2\left(p-c_{L}\right)^{2} g(p)}\left(\Phi_{L}(p)-s\right)+\alpha \frac{a_{H}}{2\left(p-c_{H}\right)^{2} g(p)}\left(\Phi_{H}(p)-s\right)
$$

and

$$
B(p) \equiv(1-\alpha) F_{L}(p)\left(\Phi_{L}(p)-s\right)+\alpha F_{H}(p)\left(\Phi_{H}(p)-s\right) .
$$

Lemma A.5. The equation $A(p)=0$ has at most one root on $Q=\left\{p: \Phi_{L}(p)>s\right\}$.

Proof. From the definition of $A(p)$ it follows that $A(p)=0$ if, and only if,

$$
\frac{\alpha\left(s-\Phi_{H}(p)\right)}{(1-\alpha)\left(\Phi_{L}(p)-s\right)}=\frac{\frac{a_{L}}{\left(p-c_{L}\right)^{2}}}{\frac{a_{H}}{\left(p-c_{H}\right)^{2}}}=\frac{\left(p-c_{H}\right)^{2}\left(\bar{p}-c_{L}\right)}{\left(p-c_{L}\right)^{2}\left(\bar{p}-c_{H}\right)} .
$$

Note that the denominator of the LHS is always positive. For all prices $p$ such that $\Phi_{H}(p) \leq s$ the numerator is also positive and the LHS is decreasing, while the RHS is increasing in $p$. 
Thus, there is at most one $p$ where $A(p)=0$. Moreover, for all $p$ such that $\Phi_{H}(p)>s$ we have $A(p)>0$, so there is no solution of $A(p)=0$ for these prices.

Lemma A.6. For all $p \in Q=\left\{p: \Phi_{L}(p)>s\right\}$ such that $A(p)>0$ we have $B(p)>0$.

Proof. Note that for all $p$ such that $\Phi_{H}(p) \geq s$ we have both $A(p)>0$ and $B(p)>0$. Now, consider prices such that $\Phi_{H}(p)<s$. Then, $A(p)>0$, if and only if,

$$
\frac{(1-\alpha)\left(\Phi_{L}(p)-s\right)}{\alpha\left(s-\Phi_{H}(p)\right)}>\frac{\frac{a_{H}}{\left(p-c_{H}\right)^{2}}}{\frac{a_{L}}{\left(p-c_{L}\right)^{2}}}=\frac{\left(p-c_{L}\right)^{2}\left(\bar{p}-c_{H}\right)}{\left(p-c_{H}\right)^{2}\left(\bar{p}-c_{L}\right)} .
$$

Similarly, $B(p)>0$, if and only if,

$$
\frac{(1-\alpha)\left(\Phi_{L}(p)-s\right)}{\alpha\left(s-\Phi_{H}(p)\right)}>\frac{F_{H}(p)}{F_{L}(p)} .
$$

The LHS of (23) and (24) are identical; as the RHS of (23) is larger than 1, while by Corollary 3 the RHS of (24) is smaller than 1, the statement follows.

Now note, that if $\rho_{L} \geq \underline{p}_{H}$ then due to Lemma 2 it follows from Lemmas A.5 and A.6 that $B(p)>0$ for all $p=\left[\rho_{L}, \bar{p}\right]$. The next lemma establishes the same result for the case where $\rho_{L}<\underline{p}_{H}$.

Lemma A.7. Suppose $\rho_{L}<\underline{p}_{H}$. Then $B(p)>0$ for all $p \in P_{(0,1)}$.

Proof. Note that

$$
B^{\prime}(p)=(1-\alpha) f_{L}(p)\left[\Phi_{L}(p)-s\right]+\alpha f_{H}(p)\left[\Phi_{H}(p)-s\right]+(1-\alpha) F_{L}(p)^{2}+\alpha F_{H}(p)^{2} .
$$

As $p \in P_{(0,1)}$ we have that the sum of the first two terms must be equal to zero due to (10). Thus, $B^{\prime}(p)>0$. Note, that $B\left(\underline{p}_{H}\right) \geq 0$ as $F_{H}\left(\underline{p}_{H}\right)=0$. Thus, as $P_{(0,1)} \subseteq \operatorname{Supp} F_{H}$ we get that $B(p)>0$ on this set.

In the proof of the Theorem, we use the fact that the system of differential equations (7) and (10) has a unique solution. To this end, we prove in the next Lemma that this is the case by applying the Pickard-Lindelof theorem.

Lemma A.8. For any $p_{1}$ the system of differential equations given by (7) and (10) with boundary values $\Phi_{i}\left(p_{1}\right), F_{i}\left(p_{1}\right), \beta\left(p_{1}\right), i=L, H$ such that

$$
\begin{aligned}
& (1-\alpha) F_{L}\left(p_{1}\right)\left(\Phi_{L}\left(p_{1}\right)-s\right)+\alpha F_{H}\left(p_{1}\right)\left(\Phi_{H}\left(p_{1}-s\right)>0\right. \\
& \beta\left(p_{1}\right) \leq 1
\end{aligned}
$$

has a unique solution in a neighborhood of $p_{1}$.

Proof. To apply the Pickard-Lindelof theorem, we need to rewrite our system in the form where the derivatives of certain functions are expressed as functions of these functions themselves. We do this in the following way: we define a function $\Phi_{i}(p)=\int_{\underline{p}}^{\bar{p}} F_{i}(p) d p$, so that $\Phi_{i}^{\prime}(p)=$ $F_{i}(p)$, and a function $z_{i}(p)=\int_{0}^{p} \frac{(1-\lambda) \beta(\bar{p})\left(\bar{p}-c_{i}\right)}{\left(x-c_{i}\right)^{2} g(x)} d x$, so that $z_{i}^{\prime}(p)=-\frac{(1-\lambda) \beta(\bar{p})\left(\bar{p}-c_{i}\right)}{\left(p-c_{i}\right)^{2} g(p)}$. Using these transformations, we can rewrite our system as 


$$
\begin{aligned}
& z_{i}^{\prime}(p)=-\frac{(1-\lambda) \beta(\bar{p})\left(\bar{p}-c_{i}\right)}{\left(p-c_{i}\right)^{2} g(p)}, i=L, H \\
& \Phi_{i}^{\prime}(p)=\frac{2 g(\bar{p})-z_{i}(p)}{2 g(p)}, i=L, H
\end{aligned}
$$

and

$$
g^{\prime}(p)=-\frac{(1-\alpha) \frac{a_{L}}{\left(p-c_{L}\right)^{2}}\left(\Phi_{L}(p)-s\right)+\alpha \frac{a_{H}}{\left(p-c_{H}\right)^{2}}\left(\Phi_{H}(p)-s\right)}{(1-\alpha)\left(z_{L}(p)-2 g(p)\right)\left(\Phi_{L}(p)-s\right)+\alpha\left(z_{H}(p)-2 g(p)\right)\left(\Phi_{H}(p)-s\right)},
$$

whenever $g(p)>\sqrt{\lambda}(\beta(p)<1)$. Note that the expression for $g^{\prime}(p)$ is equivalent to (21) and that $g^{\prime}(p)=0$ if $g(p)=\sqrt{\lambda}$.

To apply the Pickard-Lindelof theorem, we need that the RHS of this system of differential equations is Lipschitz-continuous with respect to $\left(g, z_{i}, \Phi_{i}\right), i=L, H$.

Denoting $b_{i}=-\frac{(1-\lambda) \beta(\bar{p})\left(\bar{p}-c_{i}\right)}{\left(p-c_{i}\right)^{2}}, i=L, H$, the derivatives of the vector-function representing the RHS of the system of five differential equations for $z_{L}^{\prime}, z_{H}^{\prime}, \Phi_{L}^{\prime}, \Phi_{H}^{\prime}, g^{\prime}$ with respect to $g, z_{i}, \Phi_{i}$ is summarized in the matrix

$$
\nabla=\left(\begin{array}{ccccc}
\frac{b_{L}}{g^{2}} & 0 & 0 & 0 & 0 \\
\frac{b_{H}}{g^{2}} & 0 & 0 & 0 & 0 \\
-\frac{2 g(\bar{p})-z_{L}}{2 g^{2}} & -\frac{1}{2 g} & 0 & 0 & 0 \\
-\frac{2 g(\bar{p})-z_{H}}{2 g^{2}} & 0 & -\frac{1}{2 g} & 0 & 0 \\
D_{1} & D_{2} & D_{3} & D_{4} & D_{5}
\end{array}\right)
$$

where

$$
\begin{aligned}
& D_{1}=\frac{2\left((1-\alpha) b_{L}\left(\Phi_{L}-s\right)+\alpha b_{H}\left(\Phi_{H}-s\right)\right)\left((1-\alpha)\left(\Phi_{L}-s\right)+\alpha\left(\Phi_{H}-s\right)\right)}{\left[(1-\alpha) z_{L}\left(\Phi_{L}-s\right)+\alpha z_{H}\left(\Phi_{H}-s\right)-2 g\left((1-\alpha)\left(\Phi_{L}-s\right)+\alpha\left(\Phi_{H}-s\right)\right)\right]^{2}} \\
& D_{2}=-\frac{(1-\alpha)\left(\Phi_{L}-s\right)\left[(1-\alpha) b_{L}\left(\Phi_{L}-s\right)+\alpha b_{H}\left(\Phi_{H}-s\right)\right]}{\left[(1-\alpha) z_{L}\left(\Phi_{L}-s\right)+\alpha z_{H}\left(\Phi_{H}-s\right)-2 g\left((1-\alpha)\left(\Phi_{L}-s\right)+\alpha\left(\Phi_{H}-s\right)\right)\right]^{2}} \\
& D_{3}=-\frac{\alpha\left(\Phi_{H}-s\right)\left[(1-\alpha) b_{L}\left(\Phi_{L}-s\right)+\alpha b_{H}\left(\Phi_{H}-s\right)\right]}{\left[(1-\alpha) z_{L}\left(\Phi_{L}-s\right)+\alpha z_{H}\left(\Phi_{H}-s\right)-2 g\left((1-\alpha)\left(\Phi_{L}-s\right)+\alpha\left(\Phi_{H}-s\right)\right]^{2}\right.} \\
& D_{4}=\frac{\left(2 b_{L} g-2 b_{H} g+b_{H} z_{L}-b_{L} z_{H}\right) \alpha(1-\alpha)\left(\Phi_{L}-s\right)}{\left[(1-\alpha) z_{L}\left(\Phi_{L}-s\right)+\alpha z_{H}\left(\Phi_{H}-s\right)-2 g\left((1-\alpha)\left(\Phi_{L}-s\right)+\alpha\left(\Phi_{H}-s\right)\right)\right]^{2}} \\
& D_{5}=-\frac{\left(2 b_{L} g-2 b_{H} g+b_{H} z_{L}-b_{L} z_{H}\right) \alpha(1-\alpha)\left(\Phi_{H}-s\right)}{\left[(1-\alpha) z_{L}\left(\Phi_{L}-s\right)+\alpha z_{H}\left(\Phi_{H}-s\right)-2 g\left((1-\alpha)\left(\Phi_{L}-s\right)+\alpha\left(\Phi_{H}-s\right)\right)\right]^{2}} .
\end{aligned}
$$

Due to the condition $(1-\alpha) F_{L}\left(p_{1}\right)\left(\Phi_{L}\left(p_{1}\right)-s\right)+\alpha F_{H}\left(p_{1}\right)\left(\Phi_{H}\left(p_{1}\right)-s\right)>0$ all $D_{i}$ 's are bounded and our vector-function is continuously differentiable. It is known that if a function is continuously differentiable on $\left[\underline{p}_{H}, \bar{p}\right]$, then it is Lipschitz-continuous on this interval. ${ }^{25}$ The statement of the Lemma then is an application of the Pickard-Lindelof theorem.

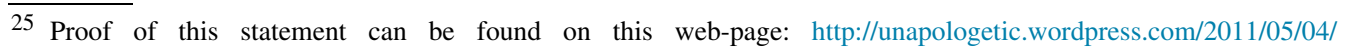
continuously-differentiable-functions-are-locally-lipschitz/. 
Note that as the five equations in the proof of Lemma A.8 are just a different representation of the system of differential equations ( 7$)$ and (10), this system with boundary conditions $F_{i}\left(p_{1}\right)$ and $\beta\left(p_{1}\right)$ also has a unique solution as long as the condition of the Lemma is satisfied. Later in the proof we show that Lemmas A.5-A.7 guarantee that the conditions of Lemma A.8 are satisfied in the relevant domains.

Theorem 1. For any values of $s, \lambda, c_{L}, c_{H}$ and $\alpha$ a NRPE as defined in Definition 2 exists. The equilibrium price distributions are characterized by (7), while non-shopper's behavior is determined by (10) whenever $0<\beta(p)<1$.

Proof. Fix some $\bar{p}>\max \left(\rho_{L}^{N U}, c_{H}+s\right)$, where $\rho_{L}^{N U}$ is the standard Stahl reservation price where there is no ex ante cost uncertainty and cost is known to be low. We first show that for any $\bar{p}$, all equilibrium conditions except $\int_{\underline{p}_{H}}^{\bar{p}} F_{H}(p) d p=s$ can be satisfied. The second part of the proof shows that this last condition can always be satisfied by an appropriate choice of $\bar{p}$. Then, by applying Lemmas A.1 and A.2 we guarantee that the distribution functions are well-defined.

We write the solution of the system of differential equations with boundary conditions $\bar{\beta}$, $F_{L}(\bar{p})=F_{H}(\bar{p})=1$ as $\hat{\beta}(p, \bar{\beta})$ and use $\beta(p)$ whenever we refer to the equilibrium stopping probability. The solution $\hat{\beta}(p, \bar{\beta})$ does not necessarily belong to $[0,1]$, while $\beta(p)$ does. Note that according to Lemmas A.6 and A.7 for any price in the support of the high-cost distribution the condition of Lemma A.8 is satisfied. Then, from Lemma A.8 the solution is unique for any $\bar{\beta}$. As solution paths cannot intersect, $\hat{\beta}(p, \bar{\beta})$ is monotone in the second argument.

Next, we argue that there exists a unique $\bar{\beta}_{0}$ such that $\max _{p \in\left[\underline{p}_{H}, \bar{p}\right]} \hat{\beta}\left(p, \bar{\beta}_{0}\right)=1$. First, Lemma A.5 together with $\hat{\beta}^{\prime}(\bar{p}, \bar{\beta})<0$ guarantee that the function $\hat{\beta}(p, \bar{\beta})$ has a unique maximum on $\left[\underline{p}_{H}, \bar{p}\right]$. Therefore, for any $\bar{\beta}$ the solution $\hat{\beta}(p, \bar{\beta})$ either attains its maximum in the interior of $\left[\underline{p}_{H}, \bar{p}\right]$ or at $\underline{p}_{H} \cdot{ }^{26}$ In the latter case $\hat{\beta}(p, \bar{\beta})$ is monotonically decreasing on $\left[\underline{p}_{H}, \bar{p}\right]$. Second, independent of whether the maximum is attained in the interior or at the lower bound, for any $b>0$ there is a value of $\bar{\beta}$ such that $\max _{p \in\left[\underline{p}_{H}, \bar{p}\right]} \hat{\beta}(p, \bar{\beta})<b$. To see this, note that if $b_{0}$ is the largest value of $\beta$ on this interval, then, as $\underline{p}_{H}>c_{H}$, we have $\pi_{L}(p)>\frac{1-\lambda}{2} b_{0}\left(p-c_{L}\right)>\frac{1-\lambda}{2} b_{0}\left(c_{H}-c_{L}\right)$. At the same time $\pi_{L}=\frac{1-\lambda}{2} \bar{\beta}\left(\bar{p}-c_{L}\right)$. Thus,

$$
b_{0}<\frac{\bar{p}-c_{L}}{c_{H}-c_{L}} \bar{\beta} \equiv b
$$

and $b$ can be chosen arbitrary small by an appropriate choice of $\bar{\beta}$. Finally, there exists a value $\bar{\beta}$ such that $\max _{p \in\left[\underline{p}_{H}, \bar{p}\right]} \hat{\beta}(p, \bar{\beta}) \geq 1$. This follows immediately from the continuous differentiability of $\hat{\beta}$ and the fact that $\hat{\beta}^{\prime}(\bar{p}, \bar{\beta})<0$. Thus, as $\hat{\beta}(p, \bar{\beta})$ is monotone in the second argument there is a unique $\bar{\beta}_{0}$ such that $\max _{p \in\left[\underline{p}_{H}, \bar{p}\right]} \hat{\beta}\left(p, \bar{\beta}_{0}\right)=1$.

Now, we show that it is always possible to choose $\bar{\beta}$ such that a least one of the four types of NRPE exist. We start with considering the case when the maximum of $\hat{\beta}\left(p, \bar{\beta}_{0}\right)$ is attained in the interior of $\left[\underline{p}_{H}, \bar{p}\right]$.

No-gap equilibrium. Consider a solution to the system of differential equations for the $\bar{\beta}_{0}$ defined above. Recall, from Lemma 2 that $p^{*}\left(\bar{\beta}_{0}\right)$ is the largest price such that $\beta(p)=1$. We consider a candidate NRPE, such that for all prices $p>p^{*}\left(\bar{\beta}_{0}\right)$ the consumer indifference condition

$\overline{26}$ With slight abuse of notation we write $\underline{p}_{H}$ instead of $\underline{p}_{H}(\bar{\beta})$. 
is satisfied and $\beta(p)=\hat{\beta}\left(p, \bar{\beta}_{0}\right)$, and for all $p \leq p^{*}\left(\bar{\beta}_{0}\right)$ we set $\beta(p)=1$. If $\rho_{L}\left(\bar{\beta}_{0}\right) \geq \underline{p}_{H}$, then it is clear from Lemmas A.1-A.3 that all conditions for a NRPE to exist (apart from $\Phi_{H}=s$ ) are satisfied.

Monopolistic gap equilibrium. Suppose then that $\rho_{L}\left(\bar{\beta}_{0}\right)<\underline{p}_{H}$. Take some price $\hat{p} \in$ $\left[\underline{p}_{H}, p^{*}\left(\bar{\beta}_{0}\right)\right]$, and construct a solution from that price $\hat{p}$ for all $p \in\left[\underline{p}_{H}, \hat{p}\right)$, using $F_{i}(\hat{p}), \Phi_{i}(\hat{p})$ and $\hat{\beta}(\hat{p})=1$ as boundary conditions. We denote this solution path as $\hat{\beta}_{\hat{p}}(p, 1)$ to make clear that this is the mathematical solution to (10) starting from $\hat{p}$ with $\hat{\beta}(\hat{p})=1$. Recall, that $p_{0}$ is the highest price smaller than $\underline{p}_{H}$ (such that $\beta\left(p_{0}\right)=1$ ) which makes the low-cost firm indifferent between charging this price and charging $\underline{p}_{H}$. To construct an NRPE, it is necessary that $p_{0}=\rho_{L}$. From Lemmas A.5 and A.6, it follows that for any $p \in\left[\underline{p}_{H}, \hat{p}\right) \frac{\partial \hat{\beta}_{\hat{p}}(p, 1)}{\partial p}>0$. Together with Lemma A.8 this implies that $\hat{\beta}_{\hat{p}}\left(\underline{p}_{H}, 1\right)$ is decreasing in $\hat{p}$ and that $p_{0}$ is continuous in

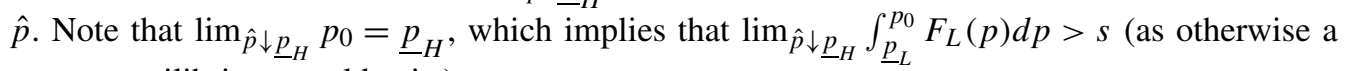
no-gap equilibrium would exist).

We derive the following two conditions from the equal profits condition (taking into account that $\left.F\left(\underline{p}_{H}\right)=F_{L}\left(p_{0}\right)\right)$

$$
\underline{p}_{L}=c_{L}+\frac{\frac{1-\lambda}{2} \bar{\beta}\left(\bar{p}-c_{L}\right)}{\frac{1+\lambda}{2}+\frac{1-\lambda}{2} \int_{\underline{p}_{H}}^{\bar{p}}(1-\beta(p)) f_{L}(p) d p}
$$

and

$$
\underline{p}_{H}-p_{0}=\frac{\left(1-\hat{\beta}_{\hat{p}}\left(\underline{p}_{H}, 1\right)\right) F_{L}\left(\underline{p}_{H}\right) \frac{1-\lambda}{2}\left(\underline{p}_{H}-c_{L}\right)}{\lambda\left(1-F_{L}\left(\underline{p}_{H}\right)\right)+\frac{1-\lambda}{2}\left(1+\int_{\underline{p}_{H}}^{\bar{p}}(1-\beta(p)) f_{L}(p) d p\right)}
$$

It is clear that $\underline{p}_{H}-p_{0}>0$ for any $\hat{\beta}_{\hat{p}}\left(\underline{p}_{H}, 1\right)<1$. As $F_{L}\left(\underline{p}_{H}\right), \int_{\underline{p}_{H}}^{\bar{p}}(1-\beta(p)) f_{L}(p) d p$ and $\int_{\underline{p}_{L}}^{p_{0}} F_{L}(p) d p$ are all continuous in $\hat{p}$ it must be the case that either

- there either exists a $\hat{p} \in\left[\underline{p}_{H}\left(\bar{\beta}_{0}\right), p^{*}\left(\bar{\beta}_{0}\right)\right]$ such that $p_{0}=\rho_{L}\left(\right.$ or $\left.\int_{\underline{p}_{L}}^{p_{0}} F_{L}(p) d p=s\right)$ and $\hat{\beta}_{\hat{p}}(p, 1) \geq 0$ for all $p \in\left[\underline{p}_{H}, p^{*}\left(\bar{\beta}_{0}\right)\right]$, meaning that there exists an NRPE;

- there exists $\hat{p}_{0} \in\left[\underline{p}_{H}\left(\bar{\beta}_{0}\right), p^{*}\left(\bar{\beta}_{0}\right)\right]$ such that $\hat{\beta}_{\hat{p}_{0}}\left(\underline{p}_{H}, 1\right)=0$ while $\int_{\underline{p}_{L}}^{p_{0}} F_{L}(p) d p>s$, and we deal with this case under the competitive gap equilibrium;

- or $\int_{\underline{p}_{L}}^{p_{0}} F_{L}(p) d p>s$ for any $\hat{p} \in\left[\underline{p}_{H}, p^{*}\right]$ and $\hat{\beta}_{\hat{p}}(p, 1)>0$ for all $p \in\left[\underline{p}_{H}, \hat{p}\right]$ and $\hat{p} \in$ $\left[\underline{p}_{H}, p^{*}\left(\bar{\beta}_{0}\right)\right]$, and we deal with this case in the regular gap equilibrium.

Regular gap equilibrium. Suppose then that for any $\hat{p} \in\left[\underline{p}_{H}, p^{*}\right], \int_{\underline{p}_{L}}^{p_{0}} F_{L}(p) d p>s$, or equivalently, $p_{0}>\rho_{L}$. Note that if $\hat{p}=p^{*}\left(\bar{\beta}_{0}\right)$ there exists a solution path $\hat{\beta}\left(p, \bar{\beta}_{0}\right)$ with $\hat{\beta}\left(p, \bar{\beta}_{0}\right) \leq 1$ and we can set $\beta(p)=\hat{\beta}\left(p, \bar{\beta}_{0}\right)$. Moreover, $\hat{\beta}(p, \bar{\beta})<1$ for any $\bar{\beta}<\bar{\beta}_{0}$. Now, note that $\lim _{\bar{\beta} \rightarrow 0} \pi_{L}=0$ and therefore $\lim _{\bar{\beta} \rightarrow 0} p_{0}=c_{L}$. As $\hat{\beta}(p, \bar{\beta})$ is continuous in both arguments, $p_{0}\left(\bar{\beta}_{0}\right)>\rho_{L}$ and $\rho_{L} \geq c_{L}+s$, there either exists $\bar{\beta}_{1}$ such that $p_{0}=\rho_{L}$ with $\hat{\beta}\left(p, \bar{\beta}_{1}\right)>0$ for all $p \in\left[\underline{p}_{H}, \bar{p}\left(\bar{\beta}_{1}\right)\right]$, meaning that the equilibrium exists, or there is a $\bar{\beta}_{2}<\bar{\beta}_{0}$ such that $\hat{\beta}\left(\underline{p}_{H}, \bar{\beta}_{2}\right)=0$ and $p_{0}\left(\bar{\beta}_{2}\right)>\rho_{L}$, and we deal with this case in the competitive gap equilibrium. 
Competitive gap equilibrium. Suppose, that either $\hat{p}_{0}$ in the monopolistic gap equilibrium or $\bar{\beta}_{2}$ in the competitive gap equilibrium exist, meaning that $\hat{\beta}\left(\underline{p}_{H}\right)=0$ and $\int_{\underline{p}_{L}}^{p_{0}} F_{L}(p) d p>s$. In both cases we take the solution on $\left[\underline{p}_{H}, \bar{p}\right]$ from the corresponding case and construct a NRPE such that $\beta(p)=0$ for all $p \in\left(\rho_{L}, \underline{p}_{H}\right]$ and $p_{0}=\rho_{L}$ and where low cost firms still choose prices in a left region of $p_{H}$, denoted by $\left[\underline{p}^{\prime}, \underline{p}_{H}\right]$. Note that as by definition $\beta\left(\underline{p}^{\prime}\right)=0$ and $\beta\left(p_{0}\right)=1$ it follows from the equal profit condition for the low cost firms that $p_{0}<\bar{p}^{\prime}$. However, by choosing $\underline{p^{\prime}}$ sufficiently low, $F_{L}\left(\underline{p}^{\prime}\right)$ can be chosen arbitrarily close to zero, which as $\int_{\underline{p}}^{\rho_{L}} F_{L}(p) d p=s$ implies that $\rho_{L}>\underline{p}^{\prime}>p_{0}$. Therefore, there must exist a $\underline{p}^{\prime}$ that $p_{0}=\rho_{L}$. This completes the proof for a given $\bar{p}$ for the case when $\beta\left(p, \bar{\beta}_{0}\right)$ reaches its maximum in the interior of $\left[\underline{p}_{H}, \bar{p}\right]$.

Consider then the case where the maximum of $\hat{\beta}\left(p, \bar{\beta}_{0}\right)$ is not attained in this interior, from Lemma A.5, it follows then that the maximum must be reached at $\underline{p}_{H}$ and $\hat{\beta}\left(\underline{p}_{H}, \bar{\beta}_{0}\right)=1$. If in this case $\rho_{L} \geq \underline{p}_{H}$, then, as previously, a no-gap equilibrium exists as we can simply set $\beta(p)=1$ for all $p<\underline{p}_{H}$. Suppose then that $\rho_{L}<\underline{p}_{H}$. If for all $\bar{\beta}<\bar{\beta}_{0}$ the maximum of $\hat{\beta}(p, \bar{\beta})$ is attained at $\underline{p}_{H}$, then a regular gap equilibrium exists with $\bar{\beta}_{1}<\bar{\beta}_{0}$ as $\hat{\beta}\left(p, \bar{\beta}_{1}\right)>0$ for all $p$ due to the monotonicity of $\hat{\beta}\left(p, \bar{\beta}_{1}\right)$ in price. If for some $\bar{\beta}<\bar{\beta}_{0}$ the maximum of $\hat{\beta}(p, \bar{\beta})$ is attained in the interior of the support of the high cost distribution, then, using our analysis in the previous case we conclude that either a regular gap or a competitive gap equilibrium exists.

We have now proved that for any fixed $\bar{p}>\max \left(c_{H}+s, \rho_{L}^{N U}\right)$ we can satisfy all equilibrium conditions apart from the fact that in an NRPE we should have $\int_{\underline{p}}^{\bar{p}} F_{H}(p)=s$. We now prove that we can always choose $\bar{p}$ such that this indifference condition is also satisfied. To do so, we first realize that as $c_{H}>c_{L}$ we have

$$
\lim _{\bar{p} \downarrow \rho_{L}^{N U}} \int_{\underline{p}_{H}}^{\bar{p}} F_{H}(p) d p<s .
$$

We next show that for sufficiently large $\bar{p}$, the other equilibrium conditions imply that

$$
\int_{\underline{p}_{H}}^{\bar{p}} F_{H}(p) d p>s .
$$

As $\int_{\underline{p}_{H}}^{\bar{p}} F_{H}(p) d p$ is continuous in $\bar{p}$, it follows then that there must be a $\bar{p}$ such that $\int_{\underline{p}_{H}}^{\bar{p}} F_{H}(p) d p=s$. Thus, the only thing to be proved is that for $\bar{p}$ sufficiently large, $\int_{\underline{p}_{H}}^{\overline{\bar{p}}} F_{H}(p) d p>s$. To this end, it follows from

$$
\pi\left(\bar{p} \mid c_{H}\right)=\frac{1-\lambda}{2}(1-\beta(\bar{p}))\left(\bar{p}-c_{H}\right)<\frac{1-\lambda}{2}\left(\bar{p}-c_{H}\right)
$$

and

$$
\pi\left(\underline{p}_{H} \mid c_{H}\right)>\frac{1+\lambda}{2}\left(\underline{p}_{H}-c_{H}\right)
$$

and the fact that a firm has to be indifferent between charging the upper and lower bound of the price distribution that 


$$
\bar{p}-\underline{p}_{H}>\frac{2 \lambda}{1+\lambda}\left(\bar{p}-c_{H}\right)
$$

Thus, the support of the mixed strategy distribution grows without bound when $\bar{p}$ becomes larger. Suppose then that $\int_{\underline{p}_{H}}^{\bar{p}} F_{H}(p) d p<s$ even for large $\bar{p}$. This would imply that for all $\epsilon>0$ there exist a large $\bar{p}$ such that $F_{H}\left(\frac{\bar{p}+\underline{p}_{H}}{2}\right)<\epsilon$. Let us then consider the profit a firm makes when setting prices $\underline{p}_{H}$ and $\left[\bar{p}+\underline{p}_{H}\right] / 2$ :

$$
\begin{aligned}
& \pi\left(\underline{p}_{H} \mid c_{H}\right)=\left[\frac{1+\lambda}{2}+\frac{1-\lambda}{2}\left(\int_{\underline{p}_{H}}^{\frac{\bar{p}+\underline{p} H}{2}}(1-\beta(\tilde{p})) f_{i}(\tilde{p}) d \tilde{p}+\int_{\frac{\bar{p}+\underline{p}_{H}}{2}}^{\bar{p}}(1-\beta(\tilde{p})) f_{i}(\tilde{p}) d \tilde{p}\right)\right] \\
& \times\left(\underline{p}_{H}-c_{H}\right) \text {, }
\end{aligned}
$$

and

$$
\begin{aligned}
\pi\left(\frac{\bar{p}+\underline{p}_{H}}{2} \mid c_{H}\right)= & {\left[\frac{1+\lambda}{2}-\left[\lambda+\frac{1-\lambda}{2}\left(1-\beta\left(\frac{\bar{p}+\underline{p}_{H}}{2}\right)\right)\right] F_{H}\left(\frac{\bar{p}+\underline{p}_{H}}{2}\right)+\right.} \\
& \left.\frac{1-\lambda}{2} \int_{\frac{\bar{p}+\underline{p}_{H}}{2}}^{\bar{p}}(1-\beta(\tilde{p})) f_{i}(\tilde{p}) d \tilde{p}\right]\left(\frac{\bar{p}+\underline{p}_{H}}{2}-c_{H}\right) .
\end{aligned}
$$

As by choosing $\bar{p}$ we can make $F_{H}\left(\frac{\bar{p}+\underline{p}_{H}}{2}\right)$ arbitrarily small and as $1-\beta(\widetilde{p})<1$, it is clear that

$$
\pi\left(\underline{p}_{H} \mid c_{H}\right)<\left[\begin{array}{r}
\left.\frac{1+\lambda}{2}+\frac{1-\lambda}{2} F_{H}\left(\frac{\bar{p}+\underline{p}_{H}}{2}\right)+\frac{1-\lambda}{2} \int_{\frac{\bar{p}+\underline{p}}{2}}^{\bar{p}}(1-\beta(\tilde{p})) f_{i}(\tilde{p}) d \tilde{p}\right] \\
\times\left(\underline{p}_{H}-c_{H}\right),
\end{array}\right.
$$

so that

$$
\begin{aligned}
& \pi\left(\frac{\bar{p}+\underline{p}_{H}}{2} \mid c_{H}\right)-\pi\left(\underline{p}_{H} \mid c_{H}\right)> \\
& {\left[\frac{1+\lambda}{2}+\frac{1-\lambda}{2} \int_{\bar{p} / 2}^{\bar{p}}(1-\beta(\tilde{p})) f_{i}(\tilde{p}) d \tilde{p}-\frac{1-\lambda}{2} F_{H}\left(\frac{\bar{p}+\underline{p}_{H}}{2}\right)\right] \frac{\bar{p}-\underline{p}_{H}}{2}-} \\
& \lambda F_{H}\left(\frac{\bar{p}+\underline{p}_{H}}{2}\right)\left(\frac{\bar{p}+\underline{p}_{H}}{2}-c_{H}\right),
\end{aligned}
$$

using (27) it follows that 


$$
\begin{aligned}
& \pi\left(\frac{\bar{p}+\underline{p}_{H}}{2} \mid c_{H}\right)-\pi\left(\underline{p}_{H} \mid c_{H}\right)> \\
& \left\{\left[\frac{1+\lambda}{2}+\frac{1-\lambda}{2} \int_{\bar{p} / 2}^{\bar{p}}(1-\beta(\tilde{p})) f_{i}(\tilde{p}) d \tilde{p}-\frac{1-\lambda}{2} F_{H}\left(\frac{\bar{p}+\underline{p}_{H}}{2}\right)\right] \frac{\lambda}{1+\lambda}\right. \\
& \left.-\lambda F_{H}\left(\frac{\bar{p}+\underline{p}_{H}}{2}\right)\right\}\left(\bar{p}-c_{H}\right),
\end{aligned}
$$

which is clearly positive for large $\bar{p}$. This implies that for large $\bar{p}$ a high cost firm cannot be indifferent over the whole support of the price distribution if $\int_{\underline{p}_{H}}^{\bar{p}} F_{H}(p) d p<s$.

\section{Appendix C. Idiosyncratic cost}

Consider the case where firms are known to have a high common cost. The low cost case is identical. The first, preliminary, but important, result is that the price distributions of the firm with low and high idiosyncratic cost cannot overlap, and that the upper bound of the low cost distribution should be no larger than the lower bound of the high cost distribution, denoted by $\underline{p}_{i H}$. To see this, suppose that both the high and low cost firm have a range of prices $p$ in the interior of their support and that they sell with probability $q(p)$. As the high cost firm has to be indifferent between charging these different prices, it follows that $q(p)\left(p-c_{i H}\right)$ equals a constant $K$ that is independent of $p$. But then the profit of the low cost firm equals $q(p)\left(p-c_{i L}\right)=K\left(p-c_{i L}\right) /\left(p-c_{i H}\right), i=L, H$, which is decreasing in $p$. As, because of the shoppers, there cannot be mass points, it follows that the distributions do not overlap.

We then derive the equilibrium price distribution functions for both types of players. The distribution function $F_{i H}(p)$ of the firm with high idiosyncratic cost has to satisfy the following

$$
\pi_{i H}(p)=\left[\lambda \gamma\left(1-F_{i H}(p)\right)+\frac{1-\lambda}{2}\right]\left(p-c_{i H}\right)=\left(\lambda \gamma+\frac{1-\lambda}{2}\right)\left(\rho_{i}-c_{i H}\right),
$$

so that

$$
\left.F_{i H}(p)\right)=1-\frac{1-\lambda}{2 \lambda \gamma} \frac{\rho_{i}-p}{p-c_{i H}} .
$$

These are the standard formulae, except for the factor $\gamma$ and the fact that the determination of the reservation price $\rho_{H}$ is different (see below). Note that as the low cost distribution is below the high cost price distribution, the high cost firm only attracts the shoppers if the other firm also has a high idiosyncratic cost component.

The distribution function $F_{i L}(p)$ of the firm with low idiosyncratic cost has to satisfy the following

$$
\pi_{i H}(p)=\left[\lambda\left\{\gamma+(1-\gamma)\left(1-F_{i L}(p)\right)\right\}+\frac{1-\lambda}{2}\right]\left(p-c_{i L}\right)=\frac{1-\lambda}{2}\left(\underline{p}_{i H}-c_{i L}\right),
$$

so that

$$
F_{i L}(p)=1-\frac{1-\lambda+2 \gamma \lambda}{2 \lambda(1-\gamma)}\left(\frac{\underline{p}_{i H}-p}{p-c_{i L}}\right) .
$$


Note here that the low cost firm always attracts the shoppers if the other firm has a high idiosyncratic cost component. Note also that if the idiosyncratic cost shock is the only uncertainty, it has to be the case that the upper bound of the low cost price distribution equals the lower bound of the high cost price distribution.

It remains to determine the reservation price $\rho_{i}$. As under idiosyncratic cost uncertainty there is no learning, consumers have to be indifferent between buying and continuing to search at the reservation price, i.e.,

$$
\rho_{i}=\gamma E_{i H}(p)+(1-\gamma) E_{i L}(p)+s,
$$

where $E_{i H}(p)$ is the expected price of a firm with idiosyncratic cost $j=L, H$. It may happen that $\rho_{i} \leq c_{i H}$, in which case the high cost firm's price distribution is degenerate and $E_{i H}(p)=c_{i H}$. Using the proof of Lemma 1 in Janssen et al. (2011), these expected prices can be written as

$$
E_{i H}(p)=\left(1-\alpha_{i H}\right) c_{i H}+\alpha_{i H} \rho_{i}, \text { and } E_{i L}(p)=\left(1-\alpha_{i L}\right) c_{i L}+\alpha_{i L} \underline{p}_{i H},
$$

respectively, where $\alpha_{i H}=\int_{0}^{1} \frac{1}{1+\frac{2 \lambda \gamma}{1-\lambda} z} d z, \alpha_{i L}=\int_{0}^{1} \frac{1}{1+\frac{2 \lambda(1-\gamma)}{1-\lambda+2 \lambda \gamma} z} d z$, and $\underline{p}_{i H}=\frac{c_{i H}+\frac{1-\lambda}{2 \lambda \gamma} \rho_{i}}{1+\frac{1-\lambda}{2 \lambda \gamma}}$. Thus, the reservation price is defined by

$$
\rho_{i}=\frac{\left[\gamma\left(1-\alpha_{i H}\right)+(1-\gamma) \frac{2 \lambda \gamma}{2 \lambda \gamma+1-\lambda} \alpha_{i L}\right] c_{i H}+(1-\gamma)\left(1-\alpha_{i L}\right) c_{i L}+s}{1-\gamma \alpha_{i H}-(1-\gamma) \frac{1-\lambda}{2 \lambda \gamma+1-\lambda} \alpha_{i L}} .
$$

This fully characterizes the equilibrium under idiosyncratic cost uncertainty only.

\section{References}

Bagwell, K., Lee, G., 2014. Number of firms and price competition. Mimeo.

Benabou, R., Gertner, R., 1993. Search with learning form prices: does increased inflationary uncertainty lead to higher prices. Rev. Econ. Stud. 60, 69-93.

Chandra, A., Tappata, M., 2011. Consumer search and dynamic price dispersion: an application to gasoline markets. Rand J. Econ. 42, 681-704.

Cho, I.K., Kreps, D.M., 1987. Signaling games and stable equilibria. Q. J. Econ. 102, 179-221.

Cho, I.K., Sobel, J., 1990. Strategic stability and uniqueness in signaling games. J. Econ. Theory 50, 381-413.

Dana, J.D., 1994. Learning in the equilibrium search model. Int. Econ. Rev. 35, 745-771.

De los Santos, B., Hortaçsu, A., Wildenbeest, M.R., 2012. Testing models of consumer search using data on web browsing and purchasing behaviour. Am. Econ. Rev. 102, 2955-2980.

Dubra, Juan, 2004. Optimism and overconfidence in search. Rev. Econ. Dyn. 7 (1), 198-218.

Duffie, D., Dworczak, P., Zhu, H., 2017. Benchmarks in search markets. J. Fin. http://dx.doi.org/10.1111/jofi.12525. In press.

Fershtman, Ch., Fishman, A., 1992. Price cycles and booms: dynamic search equilibrium. Am. Econ. Rev. 82 (5), 1221-1233.

Fishman, A., 1996. Search with learning and price adjustment dynamics. Q. J. Econ. 111, 253-268.

Honka, E., Chintagunta, Pr., 2017. Simultaneous or sequential? Search strategies in the US auto insurance industry. Mark. Sci. 36 (1), 21-42.

Janssen, M., Shelegia, S., 2015a. Consumer search and double marginalization. Am. Econ. Rev. 105 (6), $1683-1710$.

Janssen, M.C.W., Roy, S., 2010. Signaling quality through prices in an oligopoly. Games Econ. Behav. 68, $192-207$.

Janssen, M.C.W., Parakhonyak, A., 2014. Consumer search markets with costly revisits. Econ. Theory 55, $481-514$.

Janssen, M.C.W., Shelegia, S., 2015b. Beliefs and Consumer Search. Technical report. University of Vienna, Department of Economics.

Janssen, M.C.W., Pichler, P., Weidenholzer, S., 2011. Oligopolistic markets with sequential search and production cost uncertainty. Rand J. Econ. 42, 444-470.

Kalyanaram, G., Winer, R., 1995. Empirical generalizations from reference price research. Mark. Sci. 14 (3), G161-G169. 
Lauermann, S., Merzyn, W., Virag, G., 2017. Learning and price discovery in a search market. Rev. Econ. Stud. http://dx.doi.org/10.1093/restud/rdx029, forthcoming.

Mazumdar, T., Raj, S., Sinha, I., 2005. Reference price research: review and propositions. J. Mark. 69, 84-102.

McCall, J., 1970. Economics of information and job search. Q. J. Econ. 84 (1), 113-126.

Parakhonyak, A., Sobolev, A., 2015. Non-reservation price equilibrium and search without priors. Econ. J. 125 (584), 887-909.

Putler, D., 1992. Incorporating reference price effects into a theory of consumer choice. Mark. Sci. 11, $287-309$.

Rogerson, R., Shimer, R., Wright, R., 2005. Search-theoretic models of the labor market: a survey. J. Econ. Lit. 43 (4), 959-988.

Rothschild, M., 1974. Searching for the lowest price when the distribution of prices is unknown. J. Polit. Econ. 82, $689-711$.

Salop, S.C., Stiglitz, J.E., 1977. Bargains and ripoffs: a model of monopolistically competitive price dispersion. Rev. Econ. Stud. 44, 495-510.

Stahl, D.O., 1989. Oligopolistic pricing with sequential consumer search. Am. Econ. Rev. 79, 700-712.

Tappata, M., 2009. Rockets and feathers: understanding asymmetric pricing. Rand J. Econ. 40, 673-687.

Wolinsky, A., 1986. True monopolistic competition as a result of imperfect information. Q. J. Econ. 101 (3), $493-511$.

Yang, H., Ye, L., 2008. Search with learning: understanding asymmetric price adjustments. Rand J. Econ. 39 (2), $547-564$. 